\title{
Some New Hermite-Hadamard-Type Inequalities Associated with Conformable Fractional Integrals and Their Applications
}

\author{
Arshad Iqbal, ${ }^{1}$ Muhammad Adil Khan, ${ }^{1}$ Sana Ullah, ${ }^{1}$ and Yu-Ming Chu $\mathbb{1 D}^{2,3}$ \\ ${ }^{1}$ Department of Mathematics, University of Peshawar, Peshawar 25000, Pakistan \\ ${ }^{2}$ Department of Mathematics, Huzhou University, Huzhou 313000, China \\ ${ }^{3}$ School of Mathematics and Statistics, Changsha University of Science \& Technology, Changsha 410114, China \\ Correspondence should be addressed to Yu-Ming Chu; chuyuming@zjhu.edu.cn
}

Received 22 September 2019; Accepted 16 December 2019; Published 23 February 2020

Academic Editor: Kasso A. Okoudjou

Copyright ( 2020 Arshad Iqbal et al. This is an open access article distributed under the Creative Commons Attribution License, which permits unrestricted use, distribution, and reproduction in any medium, provided the original work is properly cited.

In this article, we establish some new Hermite-Hadamard-type inequalities involving the conformable fractional integrals. As applications, several inequalities for the approximation error in the midpoint formula and certain bivariate means are derived.

\section{Introduction}

Let $I \subseteq \mathbb{R}$ be an interval. Then, a real-valued function $\phi: I \longrightarrow \mathbb{R}$ is said to be convex (concave) on $I$ if the inequality

$$
\phi\left(\vartheta d_{1}+(1-\vartheta) d_{2}\right) \leq(\geq) \vartheta \phi\left(d_{1}\right)+(1-\vartheta) \phi\left(d_{2}\right),
$$

holds for all $d_{1}, d_{2} \in I$ with $d_{1} \neq d_{2}$ and $\vartheta \in[0,1]$.

It is well known that the convex functions have wide applications in pure and applied mathematics [1-27]. In particular, many remarkable inequalities and properties for the convex functions can be found in the literature [28-64]. Recently, a great deal of generalizations, extensions, and variants have been made for the convexity [65-72].

Let $d_{1}, d_{2} \in \mathbb{R}$ with $d_{1}<d_{2}$ and $\phi:\left[d_{1}, d_{2}\right] \longrightarrow \mathbb{R}$ be a convex function. Then, the classical Hermite-Hadamard inequality [73-77] states that the double inequality

$$
\phi\left(\frac{d_{1}+d_{2}}{2}\right) \leq \frac{1}{d_{2}-d_{1}} \int_{d_{1}}^{d_{2}} \phi(z) \mathrm{d} z \leq \frac{\phi\left(d_{1}\right)+\phi\left(d_{2}\right)}{2},
$$

holds.

Alomari et al. [78] and Chen and Feng [79] proved the following.

Theorem 1 (see [78]). Let $I \subseteq \mathbb{R}$ be an interval, $I^{\circ}$ be the interior of $I$, and $\phi: I^{\circ} \longrightarrow \mathbb{R}$ be a differentiable mapping. Then, the identity

$$
\begin{aligned}
\phi( & \left.\frac{d_{1}+d_{2}}{2}\right)-\frac{1}{d_{2}-d_{1}} \int_{d_{1}}^{d_{2}} \phi(z) \mathrm{d} z \\
= & \frac{d_{2}-d_{1}}{4}\left[\int_{0}^{1} \phi^{\prime}\left(\frac{\left(d_{1}+d_{2}\right) z}{2}+d_{1}(1-z)\right) z \mathrm{~d} z\right. \\
& \left.+\int_{0}^{1} \phi^{\prime}\left(\frac{\left(d_{1}+d_{2}\right)(1-z)}{2}+d_{2} z\right)(z-1) \mathrm{d} z\right],
\end{aligned}
$$

holds for $d_{1}, d_{2} \in I^{\circ}$ with $d_{1}<d_{2}$ if $\phi^{\prime} \in L\left[d_{1}, d_{2}\right]$.

Theorem 2 (see [79]). Let $I \subseteq \mathbb{R}$ be an interval, $I^{\circ}$ be the interior of $I$, and $\phi: I^{\circ} \longrightarrow \mathbb{R}$ be a differentiable mapping. Then, the identity

$$
\begin{aligned}
\phi & {\left[d_{1}+(1-\lambda) d_{2}\right]-\frac{1}{d_{2}-d_{1}} \int_{d_{1}}^{d_{2}} \phi(z) \mathrm{d} z } \\
= & \lambda^{2}\left(d_{2}-d_{1}\right)\left[\int_{0}^{1} \phi^{\prime}\left(\left(\lambda d_{1}+(1-\lambda) d_{2}\right)(1-z)+d_{2} z\right)(z-1) \mathrm{d} z\right] \\
& +(1-\lambda)^{2}\left(d_{2}-d_{1}\right)\left[\int_{0}^{1} \phi^{\prime}\left(\left(\lambda d_{1}+(1-\lambda) d_{2}\right) z+d_{1}(1-z)\right) z \mathrm{~d} z\right],
\end{aligned}
$$

holds for $d_{1}, d_{2} \in I^{\circ}$ with $d_{1}<d_{2}$ and $\lambda \in[0,1]$ if $\phi^{\prime} \in$ $L\left[d_{1}, d_{2}\right]$. 
Now, we recall the definitions of the conformable fractional derivative and integral.

Definition 1 (see [80]). Let $\phi:[0, \infty) \longrightarrow \mathbb{R}$ be a real-valued function and $\alpha \in(0,1]$. Then, the $\alpha$-order conformable fractional derivative $D_{\alpha}(\phi)(z)$ of $\phi$ at $z \in(0, \infty)$ is defined by

$$
D_{\alpha}(\phi)(z)=\lim _{\nu \longrightarrow 0} \frac{\phi\left(z+v z^{1-\alpha}\right)-\phi(z)}{v}
$$

$$
\begin{aligned}
\frac{\mathrm{d}_{\alpha}}{\mathrm{d}_{\alpha} m}\left(m^{\mu}\right) & =\mu m^{\mu-\alpha}, \\
\frac{\mathrm{d}_{\alpha}}{\mathrm{d}_{\alpha} m}(\mu) & =0 \\
\frac{\mathrm{d}_{\alpha}}{\mathrm{d}_{\alpha} m}\left(\mu_{1} \phi_{1}(m)+\mu_{2} \phi_{2}(m)\right) & =\mu_{1} \frac{\mathrm{d}_{\alpha}}{\mathrm{d}_{\alpha} m}\left(\phi_{1}(m)\right)+\mu_{2} \frac{\mathrm{d}_{\alpha}}{\mathrm{d}_{\alpha} m}\left(\phi_{2}(m)\right) \\
\frac{\mathrm{d}_{\alpha}}{\mathrm{d}_{\alpha} m}\left(\phi_{1}(m) \phi_{2}(m)\right) & =\phi_{1}(m) \frac{\mathrm{d}_{\alpha}}{\mathrm{d}_{\alpha} m}\left(\phi_{2}(m)\right)+\phi_{2}(m) \frac{\mathrm{d}_{\alpha}}{\mathrm{d}_{\alpha} m}\left(\phi_{1}(m)\right), \\
\frac{\mathrm{d}_{\alpha}}{\mathrm{d}_{\alpha} m}\left(\frac{\phi_{1}(m)}{\phi_{2}(m)}\right) & =\frac{\phi_{2}(m)\left(\mathrm{d}_{\alpha} / \mathrm{d}_{\alpha} m\right)\left(\phi_{1}(m)\right)-\phi_{1}(m)\left(\mathrm{d}_{\alpha} / \mathrm{d}_{\alpha} m\right)\left(\phi_{2}(m)\right)}{\left(\phi_{2}(m)\right)^{2}}
\end{aligned}
$$

where $\phi$ is said to be $\alpha$-differentiable at $z$ if $D_{\alpha}(\phi)(z)$ exists. In particular, $D_{\alpha}(\phi)(0)$ is defined by

$$
D_{\alpha}(\phi)(0)=\lim _{z \longrightarrow 0^{+}} D_{\alpha}(\phi)(z),
$$

and we use $\phi^{\alpha}(z)$ or $\left(\mathrm{d}_{\alpha} / \mathrm{d}_{\alpha} z\right)(\phi)$ to denote $D_{\alpha}(\phi)(z)$.

The following formulas can be derived from Definition 1 immediately.

Theorem 3. Let $\alpha \in(0,1]$ and $\phi_{1}$ and $\phi_{2}$ be two $\alpha$-differentiable real-valued functions at $m>0$. Then, one has for any constants $\mu, \mu_{1}, \mu_{2} \in \mathbb{R}$. If $\phi_{1}$ is differentiable at $\phi_{2}(m)$, then

$$
\frac{\mathrm{d}_{\alpha}}{\mathrm{d}_{\alpha} m}\left(\phi_{1}\left(\phi_{2}(m)\right)\right)=\phi_{1}^{\prime}\left(\phi_{2}(m)\right) \frac{\mathrm{d}_{\alpha}}{\mathrm{d}_{\alpha} m}\left(\phi_{2}(m)\right) .
$$

Moreover, if $\phi_{1}$ is differentiable, then

$$
\frac{\mathrm{d}_{\alpha}}{\mathrm{d}_{\alpha} m}\left(\phi_{1}(m)\right)=m^{1-\alpha} \frac{\mathrm{d}}{\mathrm{d} m}\left(\phi_{1}(m)\right) \text {. }
$$

Definition 2. Let $\alpha \in(0,1]$ and $d_{1}, d_{2} \in[0, \infty)$ with $d_{1}<d_{2}$. Then, the real-valued function $\phi_{1}:\left[d_{1}, d_{2}\right] \longrightarrow \mathbb{R}$ is said to be $\alpha$-fractional integrable if the integral

$$
\int_{d_{1}}^{d_{2}} \phi_{1}(y) \mathrm{d}_{\alpha} y=: \int_{d_{1}}^{d_{2}} \phi_{1}(y) y^{\alpha-1} \mathrm{~d} y,
$$

exists and is finite. All $\alpha$-fractional integrable functions on $\left[d_{1}, d_{2}\right]$ are denoted by $L_{\alpha}^{1}\left(\left[d_{1}, d_{2}\right]\right)$.

\section{Remark 1}

$$
I_{\alpha}^{d_{1}}\left(\phi_{1}\right)(m)=I_{1}^{d_{1}}\left(m^{\alpha-1} \phi_{1}\right)=\int_{d_{1}}^{m} \frac{\phi_{1}(y)}{y^{1-\alpha}} \mathrm{d} y,
$$

where the integral is the usual Riemann improper integral and $\alpha \in(0,1]$.

Anderson [81] established the Hermite-Hadamard inequality for the conformable fractional integral as follows. 


$$
\begin{aligned}
\phi\left(\lambda d_{1}+(1-\lambda) d_{2}\right)-\frac{\alpha}{d_{2}^{\alpha}-d_{1}^{\alpha}} \int_{d_{1}}^{d_{2}} \phi(s) \mathrm{d}_{\alpha} s \\
=\frac{\left(d_{1}-d_{2}\right)(\lambda-1)}{\left(d_{2}^{\alpha}-d_{1}^{\alpha}\right)}\left[\int_{0}^{1}\left(\left(z\left(\lambda d_{1}+(1-\lambda) d_{2}\right)+(1-z) d_{1}\right)^{2 \alpha-1}-d_{1}^{\alpha}\left(z\left(\lambda d_{1}+(1-\lambda) d_{2}\right)+(1-z) d_{1}\right)^{\alpha-1}\right)\right. \\
\left.\quad \times D_{\alpha}(\phi)\left(z\left(\lambda d_{1}+(1-\lambda) d_{2}\right)+(1-z) d_{1}\right) z^{1-\alpha} \mathrm{d}_{\alpha} z\right] \\
\quad+\frac{\left(d_{2}-d_{1}\right) \lambda}{\left(d_{2}^{\alpha}-d_{1}^{\alpha}\right)}\left[\int_{0}^{1}\left(\left(z d_{2}+(1-z)\left(\lambda d_{1}+(1-\lambda) d_{2}\right)\right)^{2 \alpha-1}-d_{2}^{\alpha}\left(z d_{2}+(1-z)\left(\lambda d_{1}+(1-\lambda) d_{2}\right)\right)^{\alpha-1}\right)\right. \\
\left.\quad \times D_{\alpha}(\phi)\left(z d_{2}+(1-z)\left(\lambda d_{1}+(1-\lambda) d_{2}\right)\right) z^{1-\alpha} d_{\alpha} z\right],
\end{aligned}
$$

holds for any $\lambda \in[0,1]$ if $D_{\alpha}(\phi) \in L_{\alpha}^{1}\left(\left[d_{1}, d_{2}\right]\right)$.

Proof. Integrating by parts, we have

$$
\begin{aligned}
& I_{1}= \int_{0}^{1}\left(\left(z\left(\lambda d_{1}+(1-\lambda) d_{2}\right)+(1-z) d_{1}\right)^{2 \alpha-1}-d_{1}^{\alpha}\left(z\left(\lambda d_{1}+(1-\lambda) d_{2}\right)+(1-z) d_{1}\right)^{\alpha-1}\right) \\
& \times D_{\alpha}(\phi)\left(z\left(\lambda d_{1}+(1-\lambda) d_{2}\right)+(1-z) d_{1}\right) z^{1-\alpha} \mathrm{d}_{\alpha} z \\
&= \int_{0}^{1}\left(\left(z\left(\lambda d_{1}+(1-\lambda) d_{2}\right)+(1-z) d_{1}\right)^{\alpha}-d_{1}^{\alpha}\right) \phi^{\prime}\left(z\left(\lambda d_{1}+(1-\lambda) d_{2}\right)+(1-z) d_{1}\right) \mathrm{d} z \\
&=\left.\left(\left(z\left(\lambda d_{1}+(1-\lambda) d_{2}\right)+(1-z) d_{1}\right)^{\alpha}-d_{1}^{\alpha}\right) \frac{\phi\left(z\left(\lambda d_{1}+(1-\lambda) d_{2}\right)+(1-z) d_{1}\right)}{\left(d_{1}-d_{2}\right)(\lambda-1)}\right|_{0} ^{1} \\
&-\alpha \int_{0}^{1}\left(z\left(\lambda d_{1}+(1-\lambda) d_{2}\right)+(1-z) d_{1}\right)^{\alpha-1} \phi\left(z\left(\lambda d_{1}+(1-\lambda) d_{2}\right)+(1-z) d_{1}\right) \mathrm{d} z \\
&=\left(\left(\lambda d_{1}+(1-\lambda) d_{2}\right)^{\alpha}-d_{1}^{\alpha}\right) \frac{\phi\left(\lambda d_{1}+(1-\lambda) d_{2}\right)}{\left(d_{1}-d_{2}\right)(\lambda-1)}-\frac{\alpha}{\left(d_{1}-d_{2}\right)(\lambda-1)} \int_{d_{1}}^{\lambda d_{1}+(1-\lambda) d_{2}} \phi(s) \mathrm{d}_{\alpha} s, \\
& I_{2}= \int_{0}^{1}\left(\left(z d_{2}+(1-z)\left(\lambda d_{1}+(1-\lambda) d_{2}\right)\right)^{2 \alpha-1}-d_{2}^{\alpha}\left(z d_{2}+(1-z)\left(\lambda d_{1}+(1-\lambda) d_{2}\right)\right)^{\alpha-1}\right) \\
& \times D_{\alpha}(\phi)\left(z d_{2}+(1-z)\left(\lambda d_{1}+(1-\lambda) d_{2}\right)\right) z^{1-\alpha} \mathrm{d}_{\alpha} z \\
&= \int_{0}^{1}\left(\left(z d_{2}+(1-z)\left(\lambda d_{1}+(1-\lambda) d_{2}\right)\right)^{\alpha}-d_{2}^{\alpha}\right) \phi^{\prime}\left(z d_{2}+(1-z)\left(\lambda d_{1}+(1-\lambda) d_{2}\right)\right) \mathrm{d} z \\
&=\left.\left(\left(z d_{2}+(1-z)\left(\lambda d_{1}+(1-\lambda) d_{2}\right)\right)^{\alpha}-d_{2}^{\alpha}\right) \frac{\phi\left(z d_{2}+(1-z)\left(\lambda d_{1}+(1-\lambda) d_{2}\right)\right)}{\left(d_{2}-d_{1}\right) \lambda}\right|_{0} ^{1} \\
&-\alpha \int_{0}^{1}\left(z d_{2}+(1-z)\left(\lambda d_{1}+(1-\lambda) d_{2}\right)\right)^{\alpha-1} \phi\left(z d_{2}+(1-z)\left(\lambda d_{1}+(1-\lambda) d_{2}\right)\right) \mathrm{d} z \\
&\left(d_{2}^{\alpha}-\left(\lambda d_{1}+(1-\lambda) d_{2}\right)^{\alpha}\right) \frac{\phi\left(\lambda d_{1}+(1-\lambda) d_{2}\right)}{\left(d_{2}-d_{1}\right) \lambda}-\frac{\alpha}{\left(d_{2}-d_{1}\right) \lambda} \int_{\lambda d_{1}+(1-\lambda) d_{2}}^{d_{2}}(s) \mathrm{d}_{\alpha} s,
\end{aligned}
$$

where we use the changes of variables $s=z\left(\lambda d_{1}+\right.$ $\left.(1-\lambda) d_{2}\right)+(1-z) d_{1}$ in $I_{1}$ and $s=z d_{2}+(1-z)\left(\lambda d_{1}+\right.$ $\left.(1-\lambda) d_{2}\right)$ in $I_{2}$. Then, multiply $I_{1}$ by $\left(\left(d_{1}-d_{2}\right)(\lambda-1)\right) /$ $\left(d_{2}^{\alpha}-d_{1}^{\alpha}\right)$ and $I_{2}$ by $\left(\left(d_{2}-d_{1}\right) \lambda\right) /\left(d_{2}^{\alpha}-d_{1}^{\alpha}\right)$ to get the desired result.
Remark 3. Let $\alpha=1$, then identity (14) reduces to (4).

Remark 4. Let $\alpha=1$ and $\lambda=1 / 2$, then identity (14) reduces to (3). 
Theorem 5. Let $\alpha \in(0,1], d_{1}, d_{2} \in(0, \infty)$ with $d_{1}<d_{2}$, and $\phi:\left[d_{1}, d_{2}\right] \longrightarrow \mathbb{R}$ be an $\alpha$-fractional differentiable function. Then, the Hermite-Hadamard-type inequality

$$
\begin{aligned}
\left|\phi\left(\lambda d_{1}+(1-\lambda) d_{2}\right)-\frac{\alpha}{d_{2}^{\alpha}-d_{1}^{\alpha}} \int_{d_{1}}^{d_{2}} \phi(s) \mathrm{d}_{\alpha} s\right| \\
\leq \frac{\left(d_{2}-d_{1}\right)(1-\lambda)}{\left(d_{2}^{\alpha}-d_{1}^{\alpha}\right)}\left[A_{1}(\alpha)\left|\phi^{\prime}\left(d_{1}\right)\right|+B_{1}(\alpha)\left|\phi^{\prime}\left(\lambda d_{1}+(1-\lambda) d_{2}\right)\right|\right] \\
\quad+\frac{\left(d_{2}-d_{1}\right) \lambda}{\left(d_{2}^{\alpha}-d_{1}^{\alpha}\right)}\left[\mathrm{A}_{2}(\alpha)\left|\phi^{\prime}\left(d_{2}\right)\right|+\mathrm{B}_{2}(\alpha)\left|\phi^{\prime}\left(\lambda d_{1}+(1-\lambda) d_{2}\right)\right|\right],
\end{aligned}
$$

holds for any $\lambda \in[0,1]$ if $D_{\alpha}(h) \in L_{\alpha}^{1}\left(\left[d_{1}, d_{2}\right]\right)$ and $\left|\phi^{\prime}\right|$ is convex on $\left[d_{1}, d_{2}\right]$, where

$$
\begin{aligned}
A_{1}(\alpha)= & \frac{1}{12}\left[\left((\lambda+1)\left(\lambda d_{1}+(1-\lambda) d_{2}\right)\left(d_{1}^{\alpha-1}+d_{2}^{\alpha-1}\right)\right)\right. \\
& \left.+\left(\lambda d_{1}\left(d_{1}^{\alpha-1}-d_{2}^{\alpha-1}\right)+d_{1}\left(d_{2}^{\alpha-1}-5 d_{1}^{\alpha-1}\right)\right)\right], \\
B_{1}(\alpha)= & \frac{\left(\lambda d_{1}+(1-\lambda) d_{2}\right)}{12}\left(3 d_{2}^{\alpha-1}(1-\lambda)+d_{1}^{\alpha-1}(1+3 \lambda)\right. \\
& \left.+d_{1}^{\alpha}(\lambda-5)\right) \\
A_{2}(\alpha)= & \frac{\lambda\left(d_{2}^{\alpha}-d_{1}^{\alpha}\right)}{6} \\
B_{2}(\alpha)= & \frac{\lambda\left(d_{2}^{\alpha}-d_{1}^{\alpha}\right)}{3} .
\end{aligned}
$$

Proof. It follows from Lemma 1 and the convexity of the functions $\psi(y)=y^{\alpha-1}$ and $\theta(y)=-y^{\alpha}$ for $y>0$ together with $\left|\phi^{\prime}\right|$ that

$$
\begin{aligned}
& \mid \phi(\left.\lambda d_{1}+(1-\lambda) d_{2}\right)-\frac{\alpha}{d_{2}^{\alpha}-d_{1}^{\alpha}} \int_{d_{1}}^{d_{2}} \phi(s) \mathrm{d}_{\alpha} s \mid \\
& \leq \frac{\left(d_{2}-d_{1}\right)(1-\lambda)}{\left(d_{2}^{\alpha}-d_{1}^{\alpha}\right)}\left[\int_{0}^{1}\left(\left(z\left(\lambda d_{1}+(1-\lambda) d_{2}\right)+(1-z) d_{1}\right)^{\alpha}-d_{1}^{\alpha}\right) \times\left|\phi^{\prime}\left(z\left(\lambda d_{1}+(1-\lambda) d_{2}\right)+(1-z) d_{1}\right)\right| \mathrm{d} z\right] \\
&+\frac{\left(d_{2}-d_{1}\right) \lambda}{\left(d_{2}^{\alpha}-d_{1}^{\alpha}\right)}\left[\int_{0}^{1}\left(d_{2}^{\alpha}-\left(z d_{2}+(1-z)\left(\lambda d_{1}+(1-\lambda) d_{2}\right)\right)^{\alpha}\right) \times\left|\phi^{\prime}\left(z d_{2}+(1-z)\left(\lambda d_{1}+(1-\lambda) d_{2}\right)\right)\right| \mathrm{d} z\right] \\
& \leq \frac{\left(d_{2}-d_{1}\right)(1-\lambda)}{\left(d_{2}^{\alpha}-d_{1}^{\alpha}\right)}\left[\int _ { 0 } ^ { 1 } \left(\left(z\left(\lambda d_{1}+(1-\lambda) d_{2}\right)+(1-z) d_{1}\right)^{\alpha-1} \times\left(z\left(\lambda d_{1}+(1-\lambda) d_{2}\right)\right.\right.\right. \\
&\left.\left.\left.+(1-z) d_{1}\right)-a^{\alpha}\right)\left|\phi^{\prime}\left(z\left(\lambda d_{1}+(1-\lambda) d_{2}\right)+(1-z) d_{1}\right)\right| \mathrm{d} z\right] \\
&+\frac{\left(d_{2}-d_{1}\right) \lambda}{\left(d_{2}^{\alpha}-d_{1}^{\alpha}\right)}\left[\int_{0}^{1}\left(d_{2}^{\alpha}-\left(z d_{2}^{\alpha}+(1-z)\left(\lambda d_{1}^{\alpha}+(1-\lambda) d_{2}^{\alpha}\right)\right)\right) \times\left|\phi^{\prime}\left(z d_{2}+(1-z)\left(\lambda d_{1}+(1-\lambda) d_{2}\right)\right)\right| \mathrm{d} z\right] \\
& \leq \frac{\left(d_{2}-d_{1}\right)(1-\lambda)}{\left(d_{2}^{\alpha}-d_{1}^{\alpha}\right)}\left[\int _ { 0 } ^ { 1 } \left(\left(z\left(\lambda d_{1}^{\alpha-1}+(1-\lambda) d_{2}^{\alpha-1}\right)+(1-z) d_{1}^{\alpha-1}\right) \times\left(z\left(\lambda d_{1}+(1-\lambda) d_{2}\right)\right.\right.\right. \\
&\left.\left.\left.\quad+(1-z) d_{1}\right)-d_{1}^{\alpha}\right)\left(z\left|\phi^{\prime}\left(\lambda d_{1}+(1-\lambda) d_{2}\right)\right|+(1-z)\left|\phi^{\prime}\left(d_{1}\right)\right|\right) \mathrm{d} z\right] \\
& \quad+\frac{\left(d_{2}-d_{1}\right) \lambda}{\left(d_{2}^{\alpha}-d_{1}^{\alpha}\right)}\left[A_{2}(\alpha)\left|\phi^{\prime}\left(d_{2}\right)\right|+B_{2}(\alpha)\left|\phi^{\prime}\left(\lambda d_{1}+(1-\lambda) d_{2}\right)\right|\right] . \\
& \quad+\frac{\left(d_{2}-d_{1}\right) \lambda}{\left(d_{2}^{\alpha}-d_{1}^{\alpha}\right)}\left[\int_{0}^{1}\left(d_{2}^{\alpha}-\left(z d_{2}^{\alpha}+(1-z)\left(\lambda d_{1}^{\alpha}+(1-\lambda) d_{2}^{\alpha}\right)\right)\right) \times\left(z\left|\phi^{\prime}\left(d_{2}\right)\right|+(1-z)\left|\phi^{\prime}\left(\lambda d_{1}+(1-\lambda) d_{2}\right)\right|\right) \mathrm{d} z\right] \\
&=
\end{aligned}
$$


Remark 5. Let $\alpha=1$. Then, inequality (16) leads to

$$
\begin{aligned}
& {\left[\phi\left(\lambda d_{1}+(1-\lambda) d_{2}\right)-\frac{\alpha}{d_{2}-d_{1}} \int_{d_{1}}^{d_{2}} \phi(s) \mathrm{d} s\right]} \\
& \leq(1-\lambda)\left[\mathrm{A}_{1}(1)\left|\phi^{\prime}\left(d_{1}\right)\right|+\mathrm{B}_{1}(1)\left|\phi^{\prime}\left(\lambda d_{1}+(1-\lambda) d_{2}\right)\right|\right] \\
& \quad+\lambda\left[\mathrm{A}_{2}(1)\left|\phi^{\prime}\left(d_{2}\right)\right|+\mathrm{B}_{2}(1)\left|\phi^{\prime}\left(\lambda d_{1}+(1-\lambda) d_{2}\right)\right|\right]
\end{aligned}
$$

where $A_{1}(\alpha), B_{1}(\alpha), A_{2}(\alpha)$, and $B_{2}(\alpha)$ are defined as in Theorem 5 .

Theorem 6. Let $\alpha \in(0,1], d_{1}, d_{2} \in(0, \infty)$ with $d_{1}<d_{2}$, and $p, q>1$ such that $1 / p+1 / q=1$ and $\phi:\left[d_{1}, d_{2}\right] \longrightarrow \mathbb{R}$ be an $\alpha$-fractional differentiable function. Then, the HermiteHadamard-type inequality

$$
\begin{gathered}
\left|\phi\left(\lambda d_{1}+(1-\lambda) d_{2}\right)-\frac{\alpha}{d_{2}^{\alpha}-d_{1}^{\alpha}} \int_{d_{1}}^{d_{2}} \phi(s) \mathrm{d}_{\alpha} s\right| \\
\leq \frac{\left(d_{2}-d_{1}\right)(1-\lambda)}{\left(d_{2}^{\alpha}-d_{1}^{\alpha}\right)}\left[\left(A_{1}(\alpha, p)\right)^{1 / p} E_{1}^{1 / q}\right] \\
+\frac{\left(d_{2}-d_{1}\right) \lambda}{\left(d_{2}^{\alpha}-d_{1}^{\alpha}\right)}\left[\left(A_{2}(\alpha, p)\right)^{1 / p} E_{2}^{1 / q}\right],
\end{gathered}
$$

$$
\begin{aligned}
& \left|\phi\left(\lambda d_{1}+(1-\lambda) d_{2}\right)-\frac{\alpha}{d_{2}^{\alpha}-d_{1}^{\alpha}} \int_{d_{1}}^{d_{2}} \phi(s) \mathrm{d}_{\alpha} s\right| \\
& \leq \frac{\left(d_{2}-d_{1}\right)(1-\lambda)}{\left(d_{2}^{\alpha}-d_{1}^{\alpha}\right)}\left[\int_{0}^{1}\left(\left(z\left(\lambda d_{1}+(1-\lambda) d_{2}\right)+(1-z) d_{1}\right)^{\alpha}-d_{1}^{\alpha}\right) \times\left|\phi^{\prime}\left(\left(\lambda d_{1}+(1-\lambda) d_{2}\right)+(1-z) d_{1}\right)\right| \mathrm{d} z\right] \\
& \quad+\frac{\left(d_{2}-d_{1}\right) \lambda}{\left(d_{2}^{\alpha}-d_{1}^{\alpha}\right)}\left[\int_{0}^{1}\left(d_{2}^{\alpha}-\left(z d_{2}+(1-z)\left(\lambda d_{1}+(1-\lambda) d_{2}\right)\right)^{\alpha}\right) \times\left|\phi^{\prime}\left(z d_{2}+(1-z)\left(\lambda d_{1}+(1-\lambda) d_{2}\right)\right)\right| \mathrm{d} z\right] \\
& \leq \frac{\left(d_{2}-d_{1}\right)(1-\lambda)}{\left(d_{2}^{\alpha}-d_{1}^{\alpha}\right)}\left[\left(\int_{0}^{1}\left(\left(z\left(\lambda d_{1}+(1-\lambda) d_{2}\right)+(1-z) d_{1}\right)^{\alpha}-d_{1}^{\alpha}\right)^{p} \mathrm{~d} z\right)^{1 / p} \times\left(\int_{0}^{1}\left|\phi^{\prime}\left(z\left(\lambda d_{1}+(1-\lambda) d_{2}\right)+(1-z) d_{1}\right)\right|^{q} \mathrm{~d} z\right)^{1 / q}\right] \\
& \quad+\frac{\left(d_{2}-d_{1}\right) \lambda}{\left(d_{2}^{\alpha}-d_{1}^{\alpha}\right)}\left[\left(\int_{0}^{1}\left(d_{2}^{\alpha}-\left(z d_{2}+(1-z)\left(\lambda d_{1}+(1-\lambda) d_{2}\right)\right)^{\alpha}\right)^{p} \mathrm{~d} z\right)^{1 / p} \times\left(\left|\phi^{\prime}\left(z d_{2}+(1-z)\left(\lambda d_{1}+(1-\lambda) d_{2}\right)\right)\right|^{q} \mathrm{~d} z\right)^{1 / q}\right] \\
& \leq \frac{\left(d_{2}-d_{1}\right)(1-\lambda)}{\left(d_{2}^{\alpha}-d_{1}^{\alpha}\right)}\left[\left(\int_{0}^{1}\left(\left(z\left(\lambda d_{1}+(1-\lambda) d_{2}\right)+(1-z) d_{1}\right)^{\alpha}-d_{1}^{\alpha}\right)^{p} \mathrm{~d} z\right)^{1 / p} \times\left(\int_{0}^{1} z\left|\phi^{\prime}\left(\lambda d_{1}+(1-\lambda) d_{2}\right)\right|^{q}+(1-z)\left|\phi^{\prime}\left(d_{1}\right)\right|^{q} \mathrm{~d} z\right)^{1 / q}\right] \\
& \quad+\frac{\left(d_{2}-d_{1}\right) \lambda}{\left(d_{2}^{\alpha}-d_{1}^{\alpha}\right)}\left[\left(\int_{0}^{1}\left(d_{2}^{\alpha}-\left(z d_{2}+(1-z)\left(\lambda d_{1}+(1-\lambda) d_{2}\right)\right)^{\alpha}\right)^{p} \mathrm{~d} z\right)^{1 / p} \times\left(z\left|\phi^{\prime}\left(d_{2}\right)\right|^{q}+(1-z)\left|\phi^{\prime}\left(\lambda d_{1}+(1-\lambda) d_{2}\right)\right|^{q} \mathrm{~d} z\right)^{1 / q}\right] \\
& =\frac{\left(d_{2}-d_{1}\right)(1-\lambda)}{\left(d_{2}^{\alpha}-d_{1}^{\alpha}\right)}\left[\left(A_{1}(\alpha, p)\right)^{1 / p}\left(E_{1}\right)^{1 / p}\right]+\frac{\left(d_{2}-d_{1}\right) \lambda}{\left(d_{2}^{\alpha}-d_{1}^{\alpha}\right)}\left[\left(A_{2}(\alpha, p)\right)^{1 / p}\left(E_{2}\right)^{1 / q}\right] .
\end{aligned}
$$

Proof. Making use of Lemma 1, the convexity of $\left|\phi^{\prime}\right|^{q}$ on $\left[d_{1}, d_{2}\right]$, Hölder inequality, and the property of the modulus, we get is valid for all $\lambda \in[0,1]$ if $D_{\alpha}(\phi) \in \mathrm{E}_{\alpha}^{1}\left(\left[d_{1}, d_{2}\right]\right)$ and $\left|\phi^{\prime}\right|^{q}$ is convex on $\left[d_{1}, d_{2}\right]$, where

$$
=\frac{1}{\lambda\left(d_{2}-d_{1}\right)} \int_{\lambda d_{1}+(1-z) d_{2}}^{d_{2}}\left(d_{2}^{\alpha}-s^{\alpha}\right)^{p} \mathrm{~d} s,
$$

$$
\begin{aligned}
& E_{1}(\alpha)=\frac{\left|\phi^{\prime}\left(\lambda d_{1}+(1-\lambda) d_{2}\right)\right|^{q}+\left|\phi^{\prime}\left(d_{1}\right)\right|^{q}}{2}, \\
& E_{2}(\alpha)=\frac{\left|\phi^{\prime}\left(d_{2}\right)\right|^{q}+\left|\phi^{\prime}\left(\lambda d_{1}+(1-\lambda) d_{2}\right)\right|^{q}}{2} .
\end{aligned}
$$

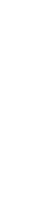

$$
A_{2}(\alpha, p)=\int_{0}^{1}\left(d_{2}^{\alpha}-\left(z d_{2}+(1-z)\left(\lambda d_{1}+(1-\lambda) d_{2}\right)\right)^{\alpha}\right)^{p} \mathrm{~d} z
$$


Remark 6. Let $\alpha=1$. Then, inequality (20) leads to

$$
\begin{aligned}
& \left|\phi\left(\lambda d_{1}+(1-\lambda) d_{2}\right)-\frac{1}{d_{2}-d_{1}} \int_{d_{1}}^{d_{2}} \phi(s) d s\right| \\
& \leq(1-\lambda)\left[\left(A_{1}(1, p)\right)^{1 / p} E_{1}^{1 / q}\right]+\lambda\left[\left(A_{2}(1, p)\right)^{1 / p} E_{2}^{1 / q}\right],
\end{aligned}
$$

where $A_{1}(\alpha, P), A_{2}(\alpha, P), E_{1}(\alpha)$, and $E_{2}(\alpha)$ are defined as in Theorem 6 .

Theorem 7. Let $\alpha \in(0,1], d_{1}, d_{2} \in(0, \infty)$ with $d_{1}<d_{2}$, $q \geq 1$, and $\phi:\left[d_{1}, d_{2}\right] \longrightarrow \mathbb{R}$ be an $\alpha$-fractional differentiable function. Then, the Hermite-Hadamard-type inequality

$$
\begin{aligned}
\left|\phi\left(\lambda d_{1}+(1-\lambda) d_{2}\right)-\frac{\alpha}{d_{2}^{\alpha}-d_{1}^{\alpha}} \int_{d_{1}}^{d_{2}} \phi(s) \mathrm{d}_{\alpha} s\right| \\
\leq \frac{\left(d_{2}-d_{1}\right)(1-\lambda)}{\left(d_{2}^{\alpha}-d_{1}^{\alpha}\right)}\left[\left(\mathrm{P}_{1}(\alpha)\right)^{1-(1 / q)}\right. \\
\left.\cdot\left(\mathrm{P}_{2}(\alpha)\left|\phi^{\prime}\left(\lambda d_{1}+(1-\lambda) d_{2}\right)\right|^{q}+\mathrm{P}_{3}(\alpha)\left|\phi^{\prime}\left(d_{1}\right)\right|^{q}\right)^{1 / q}\right] \\
+\frac{\left(d_{2}-d_{1}\right) \lambda}{\left(d_{2}^{\alpha}-d_{1}^{\alpha}\right)}\left[\left(\mathrm{Q}_{1}(\alpha)\right)^{1-(1 / q)}\right. \\
\left.\cdot\left(\mathrm{Q}_{2}(\alpha)\left|\phi^{\prime}\left(d_{2}\right)\right|^{q}+\mathrm{Q}_{3}(\alpha)\left|\phi^{\prime}\left(\lambda d_{1}+(1-\lambda) d_{2}\right)\right|^{q}\right)^{1 / q}\right]
\end{aligned}
$$

takes place for all $\lambda \in[0,1]$ if $D_{\alpha}(\phi) \in L_{\alpha}^{1}\left(\left[d_{1}, d_{2}\right]\right)$ and $\left|\phi^{\prime}\right|^{q}$ is convex on $\left[d_{1}, d_{2}\right]$, where

$$
\begin{aligned}
& P_{1}(\alpha)=\frac{1}{(\lambda-1)\left(d_{1}-d_{2}\right)}\left[\frac{\left(\left(\lambda d_{1}+(1-\lambda) d_{2}\right)^{\alpha+1}-d_{1}^{\alpha+1}\right)}{\alpha+1}-d_{1}^{\alpha}\left((\lambda-1)\left(d_{1}-d_{2}\right)\right)\right], \\
& Q_{1}(\alpha)=\frac{1}{\lambda\left(d_{2}-d_{1}\right)}\left[\frac{\left(\left(\lambda d_{1}+(1-\lambda) d_{2}\right)^{\alpha+1}-d_{2}^{\alpha+1}\right)}{\alpha+1}-d_{2}^{\alpha}\left(2 d_{2}-\lambda\left(d_{1}+d_{2}\right)\right)\right] \\
& P_{2}(\alpha)=\frac{1}{(\lambda-1)\left(d_{2}-d_{1}\right)^{2}}\left[\frac{\left(\left(\lambda d_{1}+(1-\lambda) d_{2}\right)^{\alpha+2}-d_{1}^{\alpha+2}\right)}{\alpha+2}+d_{1} \frac{\left(d_{1}^{\alpha+1}-\left(\lambda d_{1}+(1-\lambda) d_{2}\right)^{\alpha+1}\right)}{\alpha+1}\right. \\
& \left.+d_{1}^{\alpha} \frac{\left(d_{1}^{2}-\left(\lambda d_{1}+(1-\lambda) d_{2}\right)^{2}\right)}{2}+d_{1}^{\alpha+1}\left(\left(d_{1}-d_{2}\right)(1-\lambda)\right)\right] \\
& Q_{2}(\alpha)=\frac{1}{\lambda^{2}\left(d_{2}-d_{1}\right)^{2}}\left[\frac{d_{2}\left(d_{2}^{\alpha+1}-\left(\lambda d_{1}+(1-\lambda) d_{2}\right)^{\alpha+1}\right)}{\alpha+1}+\lambda\left(d_{2}-d_{1}\right) \frac{\left(\left(\lambda d_{1}+(1-\lambda) d_{2}\right)^{\alpha+1}-d_{2}^{\alpha+1}\right)}{\alpha+1}\right. \\
& \left.+\frac{\left(\left(\lambda d_{1}+(1-\lambda) d_{2}\right)^{\alpha+2}-d_{2}^{\alpha+2}\right)}{\alpha+2}+d_{2}^{\alpha+1}\left(\left(\lambda d_{1}+(1-\lambda) d_{2}\right)-d_{2}\right)+d_{2}^{\alpha} \frac{\left(d_{2}^{2}-\left(\lambda d_{1}+(1-\lambda) d_{2}\right)^{2}\right)}{2}+\lambda^{2} d_{2}^{\alpha}\left(d_{2}-d_{1}\right)^{2}\right] \\
& P_{3}(\alpha)=\frac{1}{(\lambda-1)\left(d_{2}-d_{1}\right)^{2}}\left[\frac{\left(\left(\lambda d_{1}+(1-\lambda) d_{2}\right)^{\alpha+2}-d_{1}^{\alpha+2}\right)}{\alpha+2}+d_{1} \frac{\left(d_{1}^{\alpha+1}-\left(\lambda d_{1}+(1-\lambda) d_{2}\right)^{\alpha+1}\right)}{\alpha+1}\right. \\
& \left.+d_{1}^{\alpha} \frac{\left(d_{1}^{2}-\left(\lambda d_{1}+(1-\lambda) d_{2}\right)^{2}\right)}{2}+d_{1}^{\alpha+1}\left(\left(d_{1}-d_{2}\right)(\lambda-1)\right)\right] \\
& Q_{3}(\alpha)=\frac{1}{\lambda^{2}\left(d_{2}-d_{1}\right)^{2}}\left[\frac{d_{2}\left(\left(\lambda d_{1}+(1-\lambda) d_{2}\right)^{\alpha+1}-d_{2}^{\alpha+1}\right)}{\alpha+1}+\frac{\left(d_{2}^{\alpha+2}-\left(\lambda d_{1}+(1-\lambda) d_{2}\right)^{\alpha+2}\right)}{\alpha+2}\right. \\
& \left.+d_{2}^{\alpha} \frac{\left(\left(\lambda d_{1}+(1-\lambda) d_{2}\right)^{2}-d_{2}^{2}\right)}{2}+d_{2}^{\alpha+1}\left(\lambda\left(d_{2}-d_{1}\right)\right)\right]
\end{aligned}
$$


Proof. It follows from Lemma 1, convexity of the function $\left|\phi^{\prime}\right|^{q}$, power mean inequality, and the property of the modulus that

$$
\begin{aligned}
& \left|\phi\left(\lambda d_{1}+(1-\lambda) d_{2}\right)-\frac{\alpha}{d_{2}^{\alpha}-d_{1}^{\alpha}} \int_{d_{1}}^{d_{2}} \phi(s) \mathrm{d}_{\alpha} s\right| \\
& \leq \frac{\left(d_{2}-d_{1}\right)(1-\lambda)}{\left(d_{2}^{\alpha}-d_{1}^{\alpha}\right)}\left[\int_{0}^{1}\left(\left(z\left(\lambda d_{1}+(1-\lambda) d_{2}\right)+(1-z) d_{1}\right)^{\alpha}-d_{1}^{\alpha}\right) \times\left|\phi^{\prime}\left(z\left(\lambda d_{1}+(1-\lambda) d_{2}\right)+(1-z) d_{1}\right)\right| \mathrm{d} z\right] \\
& +\frac{\left(d_{2}-d_{1}\right) \lambda}{\left(d_{2}^{\alpha}-d_{1}^{\alpha}\right)}\left[\int_{0}^{1}\left(d_{2}^{\alpha}-\left(z d_{2}+(1-z)\left(\lambda d_{1}+(1-\lambda) d_{2}\right)\right)^{\alpha}\right) \times\left|\phi^{\prime}\left(z d_{2}+(1-z)\left(\lambda d_{1}+(1-\lambda) d_{2}\right)\right)\right| \mathrm{d} z\right] \\
& \leq \frac{\left(d_{2}-d_{1}\right)(1-\lambda)}{\left(d_{2}^{\alpha}-d_{1}^{\alpha}\right)}\left[\left(\int_{0}^{1}\left(\left(z\left(\lambda d_{1}+(1-\lambda) d_{2}\right)+(1-z) d_{1}\right)^{\alpha}-d_{1}^{\alpha}\right) \mathrm{d} z\right)^{1-(1 / q)}\right. \\
& \left.\times\left(\int_{0}^{1}\left(\left(z\left(\left(\lambda d_{1}+(1-\lambda) d_{2}\right)+(1-z) d_{1}\right)^{\alpha}-d_{1}^{\alpha}\right)\left|\phi^{\prime}\left(z\left(\lambda d_{1}+(1-\lambda) d_{2}\right)+(1-z) d_{1}\right)\right|^{q}\right) \mathrm{d} z\right)^{1 / q}\right] \\
& +\frac{\left(d_{2}-d_{1}\right) \lambda}{\left(d_{2}^{\alpha}-d_{1}^{\alpha}\right)}\left[\left(\int_{0}^{1}\left(d_{2}^{\alpha}-\left(z d_{2}+(1-z)\left(\lambda d_{1}+(1-\lambda) d_{2}\right)\right)^{\alpha}\right) \mathrm{d} z\right)^{1-(1 / q)}\right. \\
& \left.\times\left(\int_{0}^{1}\left(d_{2}^{\alpha}-\left(z d_{2}+(1-z)\left(\lambda d_{1}+(1-\lambda) d_{2}\right)\right)^{\alpha}\right)\left|\phi^{\prime}\left(z d_{2}+(1-z)\left(\lambda d_{1}+(1-\lambda) d_{2}\right)\right)\right|^{q} \mathrm{~d} z\right)^{1 / q}\right] \\
& \leq \frac{\left(d_{2}-d_{1}\right)(1-\lambda)}{\left(d_{2}^{\alpha}-d_{1}^{\alpha}\right)}\left[\left(\int_{0}^{1}\left(\left(z\left(\lambda d_{1}+(1-\lambda) d_{2}\right)+(1-z) d_{1}\right)^{\alpha}-d_{1}^{\alpha}\right) \mathrm{d} z\right)^{1-(1 / q)}\right. \\
& \left.\times\left(\int_{0}^{1}\left(\left(z\left(\lambda d_{1}+(1-\lambda) d_{2}\right)+(1-z) d_{1}\right)^{\alpha}-d_{1}^{\alpha}\right)\left(z\left|\phi^{\prime}\left(\lambda d_{1}+(1-\lambda) d_{2}\right)\right|^{q}+(1-z)\left|\phi^{\prime}\left(d_{1}\right)\right|^{q} \mathrm{~d} z\right)\right)^{1 / q}\right] \\
& +\frac{\left(d_{2}-d_{1}\right) \lambda}{\left(d_{2}^{\alpha}-d_{1}^{\alpha}\right)}\left[\left(\int_{0}^{1}\left(d_{2}^{\alpha}-\left(z d_{2}+(1-z)\left(\lambda d_{1}+(1-\lambda) d_{2}\right)\right)^{\alpha}\right) \mathrm{d} z\right)^{1-(1 / q)}\right. \\
& \left.\times\left(\int_{0}^{1}\left(d_{2}^{\alpha}-\left(z d_{2}+(1-z)\left(\lambda d_{1}+(1-\lambda) d_{2}\right)\right)^{\alpha}\right)\left(z\left|\phi^{\prime}\left(d_{2}\right)\right|^{q}+(1-z)\left|\phi^{\prime}\left(\lambda d_{1}+(1-\lambda) d_{2}\right)\right|^{q} \mathrm{~d} z\right)\right)^{1 / q}\right] \\
& =\frac{\left(d_{2}-d_{1}\right)(1-\lambda)}{\left(d_{2}^{\alpha}-d_{1}^{\alpha}\right)}\left[\left(P_{1}(\alpha)\right)^{1-(1 / q)}\left(P_{2}(\alpha)\left|\phi^{\prime}\left(\lambda d_{1}+(1-\lambda) d_{2}\right)\right|^{q}+P_{3}(\alpha)\left|\phi^{\prime}\left(d_{1}\right)\right|^{q}\right)^{1 / q}\right] \\
& +\frac{\left(d_{2}-d_{1}\right) \lambda}{\left(d_{2}^{\alpha}-d_{1}^{\alpha}\right)}\left[\left(Q_{1}(\alpha)\right)^{1-(1 / q)}\left(Q_{2}(\alpha)\left|\phi^{\prime}\left(d_{2}\right)\right|^{q}+Q_{3}(\alpha)\left|\phi^{\prime}\left(\lambda d_{1}+(1-\lambda) d_{2}\right)\right|^{q}\right)^{1 / q}\right] .
\end{aligned}
$$

Remark 7. Let $\alpha=1$. Then, from inequality (24), we get

$$
\begin{aligned}
\mid \phi( & \left.\lambda d_{1}+(1-\lambda) d_{2}\right)-\frac{1}{d_{2}-d_{1}} \int_{d_{1}}^{d_{2}} \phi(s) \mathrm{d} s \mid \\
\leq & (1-\lambda)\left[\left(P_{1}(1)\right)^{1-(1 / q)}\left(P_{2}(1)\left|\phi^{\prime}\left(\lambda d_{1}+(1-\lambda) d_{2}\right)\right|^{q}+P_{3}(1)\left|\phi^{\prime}\left(d_{1}\right)\right|^{q}\right)^{1 / q}\right] \\
& +\lambda\left[\left(Q_{1}(1)\right)^{1-(1 / q)}\left(Q_{2}(1)\left|\phi^{\prime}\left(d_{2}\right)\right|^{q}+Q_{3}(1)\left|\phi^{\prime}\left(\lambda d_{1}+(1-\lambda) d_{2}\right)\right|^{q}\right)^{1 / q}\right],
\end{aligned}
$$


where $P_{1}(\alpha), P_{2}(\alpha), P_{3}(\alpha), Q_{1}(\alpha), Q_{2}(\alpha)$, and $Q_{3}(\alpha)$ are defined as in Theorem 7 .
Theorem 8. Let $\alpha \in(0,1], d_{1}, d_{2} \in(0, \infty)$ with $d_{1}<d_{2}$, $q \geq 1$, and $\phi:\left[d_{1}, d_{2}\right] \longrightarrow \mathbb{R}$ be an $\alpha$-fractional differentiable function. Then, the Hermite-Hadamard-type inequality

$$
\begin{aligned}
& \left|\phi\left(\lambda d_{1}+(1-\lambda) d_{2}\right)-\frac{\alpha}{d_{2}^{\alpha}-d_{1}^{\alpha}} \int_{d_{1}}^{d_{2}} \phi(s) \mathrm{d}_{\alpha} s\right| \\
& \leq \frac{\left(d_{2}-d_{1}\right)(1-\lambda)}{\left(d_{2}^{\alpha}-d_{1}^{\alpha}\right)}\left[\left(\left(\mathrm{M}_{1}(\alpha)\right)^{1-(1 / q)}\left(\mathrm{M}_{2}(\alpha)\left|\phi^{\prime}\left(\lambda d_{1}+(1-\lambda) d_{2}\right)\right|^{q}+\mathrm{M}_{3}(\alpha)\left|\phi^{\prime}\left(d_{1}\right)\right|^{q}\right)\right)^{1 / q}\right] \\
& \quad+\frac{\left(d_{2}-d_{1}\right) \lambda}{\left(d_{2}^{\alpha}-d_{1}^{\alpha}\right)}\left[\left(N_{1}(\alpha)\right)^{1-(1 / q)}\left(N_{2}(\alpha)\left|\phi^{\prime}\left(d_{2}\right)\right|^{q}+N_{3}(\alpha)\left|\phi^{\prime}\left(\lambda d_{1}+(1-\lambda) d_{2}\right)\right|^{q}\right)^{1 / q}\right],
\end{aligned}
$$

takes place for all $\lambda \in[0,1]$ if $D_{\alpha}(\phi) \in L_{\alpha}^{1}\left(\left[d_{1}, d_{2}\right]\right)$ and $\left|\phi^{\prime}\right|^{q}$

is convex on $\left[d_{1}, d_{2}\right]$, where

$$
\begin{aligned}
& M_{1}(\alpha)=\left[\frac{d_{1}^{\alpha}\left(\lambda^{2}+\lambda-2\right)+d_{2}^{\alpha}(\lambda-1)^{2}}{3}-\frac{d_{2} d_{1}^{\alpha-1}\left(2 \lambda^{2}+\lambda-3\right)+d_{1} d_{2}^{\alpha-1}\left(2 \lambda^{2}-\lambda-1\right)}{6}\right], \\
& N_{1}(\alpha)=\frac{\lambda\left(d_{2}^{\alpha}-d_{1}^{\alpha}\right)}{2} \\
& M_{2}(\alpha)=\frac{(\lambda-1)\left(d_{1}^{\alpha}-d_{2}^{\alpha}\right)}{3}
\end{aligned}
$$

Proof. From Lemma 1 and convexity of $\left|\phi^{\prime}\right|^{q}$, we get

$$
\begin{aligned}
\mid \phi & \left.\lambda d_{1}+(1-\lambda) d_{2}\right)-\frac{\alpha}{d_{2}^{\alpha}-d_{1}^{\alpha}} \int_{d_{1}}^{d_{2}} \phi(s) \mathrm{d}_{\alpha} s \mid \\
\leq & \frac{\left(d_{2}-d_{1}\right)(1-\lambda)}{\left(d_{2}^{\alpha}-d_{1}^{\alpha}\right)}\left[\int_{0}^{1}\left(\left(z\left(\lambda d_{1}+(1-\lambda) d_{2}\right)+(1-z) d_{1}\right)^{\alpha}-d_{1}^{\alpha}\right) \times\left|\phi^{\prime}\left(z\left(\lambda d_{1}+(1-\lambda) d_{2}\right)+(1-z) d_{1}\right)\right| \mathrm{d} z\right] \\
& +\frac{\left(d_{2}-d_{1}\right) \lambda}{\left(d_{2}^{\alpha}-d_{1}^{\alpha}\right)}\left[\int_{0}^{1}\left(d_{2}^{\alpha}-\left(z d_{2}+(1-z)\left(\lambda d_{1}+(1-\lambda) d_{2}\right)\right)^{\alpha}\right) \times\left|\phi^{\prime}\left(z d_{2}+(1-z)\left(\lambda d_{1}+(1-\lambda) d_{2}\right)\right)\right| \mathrm{d} z\right] \\
\leq & \frac{\left(d_{2}-d_{1}\right)(1-\lambda)}{\left(d_{2}^{\alpha}-d_{1}^{\alpha}\right)}\left[\left(\int_{0}^{1}\left(\left(z\left(\lambda d_{1}+(1-\lambda) d_{2}\right)+(1-z) d_{1}\right)^{\alpha}-d_{1}^{\alpha}\right) \mathrm{d} z\right)^{1-(1 / q)}\right. \\
& \left.\times\left(\int_{0}^{1}\left(\left(z\left(\lambda d_{1}+(1-\lambda) d_{2}\right)+(1-z) d_{1}\right)^{\alpha}-d_{1}^{\alpha}\right)\left|\phi^{\prime}\left(z\left(\lambda d_{1}+(1-\lambda) d_{2}\right)+(1-z) d_{1}\right)\right|^{q} \mathrm{~d} z\right)^{1 / q}\right] \\
& +\frac{\left(d_{2}-d_{1}\right) \lambda}{\left(d_{2}^{\alpha}-d_{1}^{\alpha}\right)}\left[\left(\int_{0}^{1}\left(d_{2}^{\alpha}-\left(z d_{2}+(1-z)\left(\lambda d_{1}+(1-\lambda) d_{2}\right)\right)^{\alpha}\right) \mathrm{d} z\right)^{1-(1 / q)}\right. \\
& \left.\times\left(\int_{0}^{1}\left(d_{2}^{\alpha}-\left(z d_{2}+(1-z)\left(\lambda d_{1}+(1-\lambda) d_{2}\right)\right)^{\alpha}\right)\left|\phi^{\prime}\left(z d_{2}+(1-z)\left(\lambda d_{1}+(1-\lambda) d_{2} s\right)\right)\right|^{q} \mathrm{~d} z\right)^{1 / q}\right]
\end{aligned}
$$




$$
\begin{aligned}
& \leq \frac{\left(d_{2}-d_{1}\right)(1-\lambda)}{\left(d_{2}^{\alpha}-d_{1}^{\alpha}\right)}\left[\left(\int_{0}^{1}\left(\left(z\left(\lambda d_{1}+(1-\lambda) d_{2}\right)+(1-z) d_{1}\right)^{\alpha-1} \times\left(z\left(\lambda d_{1}+(1-\lambda) d_{2}\right)+(1-z) d_{1}\right)-d_{1}^{\alpha}\right) \mathrm{d} z\right)^{1-(1 / q)}\right. \\
& \left.\times \int_{0}^{1}\left(\left(z\left(\lambda d_{1}+(1-\lambda) d_{2}\right)+(1-z) d_{1}\right)^{\alpha}-d_{1}^{\alpha}\right)\left(z\left|\phi^{\prime}\left(\lambda d_{1}+(1-\lambda) d_{2}\right)\right|^{q}+(1-z)\left|\phi^{\prime}\left(d_{1}\right)\right|^{q} \mathrm{~d} z\right)^{1 / q}\right] \\
& +\frac{\left(d_{2}-d_{1}\right) \lambda}{\left(d_{2}^{\alpha}-d_{1}^{\alpha}\right)}\left[\left(\int_{0}^{1}\left(d_{2}^{\alpha}-\left(z d_{2}+(1-z)\left(\lambda d_{1}+(1-\lambda) d_{2}\right)\right)^{\alpha}\right) \mathrm{d} z\right)^{1-(1 / q)}\right. \\
& \left.\times\left(\int_{0}^{1}\left(d_{2}^{\alpha}-\left(z d_{2}+(1-z)\left(\lambda d_{1}+(1-\lambda) d_{2}\right)\right)^{\alpha}\right)\left(z\left|\phi^{\prime}\left(d_{2}\right)\right|^{q}+(1-z)\left|\phi^{\prime}\left(\lambda d_{1}+(1-\lambda) d_{2}\right)\right|^{q} \mathrm{~d} z\right)\right)^{1 / q}\right] \\
& \leq \frac{\left(d_{2}-d_{1}\right)(1-\lambda)}{\left(d_{2}^{\alpha}-d_{1}^{\alpha}\right)}\left[\left(\int_{0}^{1}\left(\left(z\left(\lambda d_{1}^{\alpha-1}+(1-\lambda) d_{2}^{\alpha-1}\right)+(1-z) d_{1}^{\alpha-1}\right)\right) \times\left(z\left(\lambda d_{1}+(1-\lambda) d_{2}\right)+(1-z) d_{1}\right)-d_{1}^{\alpha}\right) \mathrm{d} z\right)^{1-(1 / q)} \\
& \left.\times\left(\int_{0}^{1}\left(\left(z\left(\lambda d_{1}^{\alpha}+(1-\lambda) d_{2}^{\alpha}\right)+(1-z) d_{1}^{\alpha}\right)-d_{1}^{\alpha}\right)\left(z\left|\phi^{\prime}\left(\lambda d_{1}+(1-\lambda) b\right)\right|^{q}+(1-z)\left|\phi^{\prime}\left(d_{1}\right)\right|^{q} \mathrm{~d} z\right)\right)^{1 / q}\right] \\
& +\frac{\left(d_{2}-d_{1}\right) \lambda}{\left(d_{2}^{\alpha}-d_{1}^{\alpha}\right)}\left[\left(\int_{0}^{1}\left(d_{2}^{\alpha}-\left(z d_{2}^{\alpha}+(1-z)\left(\lambda d_{1}^{\alpha}+(1-\lambda) d_{2}^{\alpha}\right)\right)\right) \mathrm{d} z\right)^{1-(1 / q)}\right. \\
& \left.\times\left(\int_{0}^{1}\left(d_{2}^{\alpha}-\left(z d_{2}^{\alpha}+(1-z)\left(\lambda d_{1}^{\alpha}+(1-\lambda) d_{2}^{\alpha}\right)\right)\right)\left(z\left|\phi^{\prime}\left(d_{2}\right)\right|^{q}+(1-z)\left|\phi^{\prime}\left(\lambda d_{1}+(1-\lambda) d_{2}\right)\right|^{q} \mathrm{~d} z\right)\right)^{1 / q}\right] \\
& =\frac{\left(d_{2}-d_{1}\right)(1-\lambda)}{\left(d_{2}^{\alpha}-d_{1}^{\alpha}\right)}\left[\left(M_{1}(\alpha)\right)^{1-(1 / q)}\left(M_{2}(\alpha)\left|\phi^{\prime}\left(\lambda d_{1}+(1-\lambda) d_{2}\right)\right|^{q}+M_{3}(\alpha)\left|\phi^{\prime}\left(d_{1}\right)\right|^{q}\right)^{1 / q}\right] \\
& +\frac{\left(d_{2}-d_{1}\right) \lambda}{\left(d_{2}^{\alpha}-d_{1}^{\alpha}\right)}\left[\left(N_{1}(\alpha)\right)^{1-(1 / q)}\left(N_{2}(\alpha)\left|\phi^{\prime}\left(d_{2}\right)\right|^{q}+N_{3}(\alpha)\left|\phi^{\prime}\left(\lambda d_{1}+(1-\lambda) d_{2}\right)\right|^{q}\right)^{1 / q}\right] .
\end{aligned}
$$

Remark 8 . Let $\alpha=1$. Then, inequality (28) becomes

$$
\begin{aligned}
& \left|\phi\left(\lambda d_{1}+(1-\lambda) d_{2}\right)-\frac{1}{d_{2}-d_{1}} \int_{d_{1}}^{d_{2}} \phi(s) \mathrm{d} s\right| \\
& \leq(1-\lambda)\left[\left(M_{1}(1)\right)^{1-(1 / q)}\left(M_{2}(1)\left|\phi^{\prime}\left(\lambda d_{1}+(1-\lambda) d_{2}\right)\right|^{q}+M_{3}(1)\left|\phi^{\prime}\left(d_{1}\right)\right|^{q}\right)^{1 / q}\right] \\
& \quad+\lambda\left[\left(N_{1}(1)\right)^{1-(1 / q)}\left(N_{2}(1)\left|\phi^{\prime}\left(d_{2}\right)\right|^{q}+N_{3}(1)\left|\phi^{\prime}\left(\lambda d_{1}+(1-\lambda) d_{2}\right)\right|^{q}\right)^{1 / q}\right],
\end{aligned}
$$

where $M_{1}(\alpha), M_{2}(\alpha), M_{3}(\alpha), N_{1}(\alpha), N_{2}(\alpha)$, and $N_{3}(\alpha)$ are defined as in Theorem 8 .
Theorem 9. Let $\alpha \in(0,1], d_{1}, d_{2} \in(0, \infty)$ with $d_{1}<d_{2}$, $q>1$, and $\phi:\left[d_{1}, d_{2}\right] \longrightarrow \mathbb{R}$ be an $\alpha$-fractional differentiable function. Then, the Hermite-Hadamard-type inequality

$$
\begin{aligned}
& \left|\phi\left(\lambda d_{1}+(1-\lambda) d_{2}\right)-\frac{\alpha}{d_{2}^{\alpha}-d_{1}^{\alpha}} \int_{d_{1}}^{d_{2}} \phi(s) \mathrm{d}_{\alpha} s\right| \\
& \quad \leq \frac{\left(d_{2}-d_{1}\right)(1-\lambda)}{\left(d_{2}^{\alpha}-d_{1}^{\alpha}\right)}\left[A_{1}(\alpha) \phi^{\prime}\left(\frac{C_{1}(\alpha)}{A_{1}(\alpha)}\right)\right]+\frac{\left(d_{2}-d_{1}\right) \lambda}{\left(d_{2}^{\alpha}-d_{1}^{\alpha}\right)}\left[B_{1}(\alpha) \phi^{\prime}\left(\frac{C_{2}(\alpha)}{B_{1}(\alpha)}\right)\right],
\end{aligned}
$$


holds for any $\lambda \in[0,1]$ if $D_{\alpha}(\phi) \in L_{\alpha}^{1}\left(\left[d_{1}, d_{2}\right]\right)$ and $\left|\phi^{\prime}\right|^{q}$ is concave on $\left[d_{1}, d_{2}\right]$, where

$$
\begin{aligned}
& A_{1}(\alpha)=\frac{1}{\left(d_{1}-d_{2}\right)(\lambda-1)}\left[\frac{\left(\left(\lambda d_{1}+(1-\lambda) d_{2}\right)^{\alpha+1}-d_{1}^{\alpha+1}\right)}{\alpha+1}+d_{1}^{\alpha}\left((\lambda-1)\left(d_{2}-d_{1}\right)\right)\right], \\
& B_{1}(\alpha)=\frac{1}{\lambda\left(d_{2}-d_{1}\right)}\left[d_{2}^{\alpha}\left(\lambda\left(d_{2}-d_{1}\right)\right)+\frac{\left(\lambda d_{1}+(1-\lambda) d_{2}\right)^{\alpha+1}-d_{2}^{\alpha+1}}{\alpha+1}\right], \\
& C_{1}(\alpha)=\frac{1}{\left(d_{1}-d_{2}\right)(\lambda-1)}\left[\frac{\left(\left(\lambda d_{1}+(1-\lambda) d_{2}\right)^{\alpha+2}-d_{1}^{\alpha+2}\right)}{\alpha+2}+d_{1}^{\alpha} \frac{\left(d_{1}^{2}-\left(\lambda d_{1}+(1-\lambda) d_{2}\right)^{2}\right)}{2}\right], \\
& C_{2}(\alpha)=\frac{1}{\lambda\left(d_{2}-d_{1}\right)}\left[\frac{\left(\left(\lambda d_{1}+(1-\lambda) d_{2}\right)^{\alpha+2}-d_{2}^{\alpha+2}\right)}{\alpha+2}+d_{2}^{\alpha} \frac{\left(d_{2}^{2}-\left(\lambda d_{1}+(1-\lambda) d_{2}\right)^{2}\right)}{2}\right] .
\end{aligned}
$$

Proof. Let $\gamma \in[0,1]$. Then, the power mean inequality and which shows that $\left|\phi^{\prime}\right|$ is also concave. the concavity of $\left|\phi^{\prime}\right|^{q}$ lead to the conclusion that

$$
\begin{aligned}
& \left(\gamma\left|\phi^{\prime}\left(d_{1}\right)\right|+(1-\gamma)\left|\phi^{\prime}\left(d_{2}\right)\right|\right)^{q} \\
& \leq \gamma\left|\phi^{\prime}\left(d_{1}\right)\right|^{q}+(1-\gamma)\left|\phi^{\prime}\left(d_{2}\right)\right|^{q} \\
& \leq\left|\phi^{\prime}\left(\gamma d_{1}+(1-\gamma) d_{2}\right)\right|^{q},
\end{aligned}
$$

Making use of Lemma 1, we get

$$
\begin{aligned}
& \left|\phi\left(\lambda d_{1}+(1-\lambda) d_{2}\right)-\frac{\alpha}{d_{2}^{\alpha}-d_{1}^{\alpha}} \int_{d_{1}}^{d_{2}} \phi(s) \mathrm{d}_{\alpha} s\right| \\
& \leq \frac{\left(d_{2}-d_{1}\right)(1-\lambda)}{\left(d_{2}^{\alpha}-d_{1}^{\alpha}\right)}\left[\int_{0}^{1}\left(\left(z\left(\lambda d_{1}+(1-\lambda) d_{2}\right)+(1-z) d_{1}\right)^{\alpha}-d_{1}^{\alpha}\right) \times\left|\phi^{\prime}\left(\left(\lambda d_{1}+(1-\lambda) d_{2}\right)+(1-z) d_{1}\right)\right| \mathrm{d} z\right] \\
& \quad+\frac{\left(d_{2}-d_{1}\right) \lambda}{\left(d_{2}^{\alpha}-d_{1}^{\alpha}\right)}\left[\int_{0}^{1}\left(d_{2}^{\alpha}-\left(z d_{2}+(1-z)\left(\lambda d_{1}+(1-\lambda) d_{2}\right)\right)^{\alpha}\right) \times\left|\phi^{\prime}\left(z d_{2}+(1-z)\left(\lambda d_{1}+(1-\lambda) d_{2}\right)\right)\right| \mathrm{d} z\right] .
\end{aligned}
$$

Applying Jensen integral inequality, we have

$$
\begin{aligned}
& \int_{0}^{1}\left(\left(z\left(\lambda d_{1}+(1-\lambda) d_{2}\right)+(1-z) d_{1}\right)^{\alpha}-d_{1}^{\alpha}\right)\left|\phi^{\prime}\left(\left(\lambda d_{1}+(1-\lambda) d_{2}\right)+(1-z) d_{1}\right)\right| \mathrm{d} z \\
& \leq\left(\int_{0}^{1}\left(\left(z\left(\lambda d_{1}+(1-\lambda) d_{2}\right)+(1-z) d_{1}\right)^{\alpha}-d_{1}^{\alpha}\right) \mathrm{d} z\right) \\
& \quad \times \phi^{\prime}\left(\frac{\left(\int_{0}^{1}\left(\left(z\left(\lambda d_{1}+(1-\lambda) d_{2}\right)+(1-z) d_{1}\right)^{\alpha}-d_{1}^{\alpha}\right)\left(z\left(\lambda d_{1}+(1-\lambda) d_{2}\right)+(1-z) d_{1}\right) \mathrm{d} z\right)}{\int_{0}^{1}\left(\left(z\left(\lambda d_{1}+(1-\lambda) d_{2}\right)+(1-z) d_{1}\right)^{\alpha}-d_{1}^{\alpha}\right) \mathrm{d} z}\right) \\
& =A_{1}(\alpha) \phi^{\prime}\left(\frac{C_{1}(\alpha)}{A_{1}(\alpha)}\right) .
\end{aligned}
$$


Similarly, one has

$$
\begin{aligned}
& \int_{0}^{1}\left(d_{2}^{\alpha}-\left(z d_{2}+(1-z)\left(\lambda d_{1}+(1-\lambda) d_{2}\right)\right)^{\alpha}\right)\left|\phi^{\prime}\left(d_{2}^{\alpha}-\left(z d_{2}+(1-z)\left(\lambda d_{1}+(1-\lambda) d_{2}\right)\right)\right)\right| \mathrm{d} z \\
& \leq\left(\int_{0}^{1}\left(d_{2}^{\alpha}-\left(z d_{2}+(1-z)\left(\lambda d_{1}+(1-\lambda) d_{2}\right)\right)^{\alpha}\right) \mathrm{d} z\right) \\
& \quad \times \phi^{\prime}\left(\frac{\int_{0}^{1}\left(d_{2}^{\alpha}-\left(z d_{2}+(1-z)\left(\lambda d_{1}+(1-\lambda) d_{2}\right)\right)^{\alpha}\right)\left(\left(z d_{2}+(1-z)\left(\lambda d_{1}+(1-\lambda) d_{2}\right)\right) \mathrm{d} z\right.}{\int_{0}^{1}\left(d_{2}^{\alpha}-\left(z d_{2}+(1-z)\left(\lambda d_{1}+(1-\lambda) d_{2}\right)\right)^{\alpha}\right) \mathrm{d} z}\right) \\
& =B_{1}(\alpha) \phi^{\prime}\left(\frac{C_{2}(\alpha)}{B_{1}(\alpha)}\right) .
\end{aligned}
$$

Remark 9. Let $\alpha=1$. Then, inequality (32) gives

$$
\begin{aligned}
& \left|\phi\left(\lambda d_{1}+(1-\lambda) d_{2}\right)-\frac{1}{d_{2}-d_{1}} \int_{d_{1}}^{d_{2}} \phi(s) \mathrm{d} s\right| \\
& \quad \leq(1-\lambda)\left[A_{1}(1) \phi^{\prime}\left(\frac{C_{1}(1)}{A_{1}(1)}\right)\right]+\lambda\left[B_{1}(1) \phi^{\prime}\left(\frac{C_{2}(1)}{B_{1}(1)}\right)\right],
\end{aligned}
$$

where $A_{1}(\alpha), C_{1}(\alpha), B_{1}(\alpha)$, and $C_{2}(\alpha)$ are defined as in Theorem 9 .

Theorem 10. Let $\alpha \in(0,1], d_{1}, d_{2} \in(0, \infty)$ with $d_{1}<d_{2}$, and $\phi:\left[d_{1}, d_{2}\right] \longrightarrow \mathbb{R}$ be an $\alpha$-fractional differentiable function. Then, the Hermite-Hadamard-type inequality

$$
\begin{gathered}
\left|\phi\left(\lambda d_{1}+(1-\lambda) d_{2}\right)-\frac{\alpha}{d_{2}^{\alpha}-d_{1}^{\alpha}} \int_{d_{1}}^{d_{2}} \phi(s) \mathrm{d}_{\alpha} s\right| \\
\leq \frac{\left(d_{2}-d_{1}\right)(1-\lambda)}{\left(d_{2}^{\alpha}-d_{1}^{\alpha}\right)}\left[A_{1}(\alpha)\left|\phi^{\prime}\left(d_{1}\right)\right|+B_{1}(\alpha)\left|\phi^{\prime}\left(\lambda d_{1}+(1-\lambda) d_{2}\right)\right|\right] \\
+\frac{\left(d_{2}-d_{1}\right) \lambda}{\left(d_{2}^{\alpha}-d_{1}^{\alpha}\right)}\left[A_{2}(\alpha)\left|\phi^{\prime}\left(d_{2}\right)\right|+B_{2}(\alpha)\left|\phi^{\prime}\left(\lambda d_{1}+(1-\lambda) d_{2}\right)\right|\right],
\end{gathered}
$$

holds for all $\lambda \in[0,1]$ if $D_{\alpha}(\phi) \in L_{\alpha}^{1}\left(\left[d_{1}, d_{2}\right]\right)$ and $\left|\phi^{\prime}\right|$ is convex on $\left[d_{1}, d_{2}\right]$, where $A_{1}(\alpha), B_{1}(\alpha), A_{2}(\alpha)$, and $B_{2}(\alpha)$ are defined as in Theorem 5.

Proof. It follows from Lemma 1 and the convexity of $\left|\phi^{\prime}\right|$ that

$$
\begin{aligned}
\mid \phi & \left(\lambda d_{1}+(1-\lambda) d_{2}\right)-\frac{\alpha}{d_{2}^{\alpha}-d_{1}^{\alpha}} \int_{d_{1}}^{d_{2}} \phi(s) \mathrm{d}_{\alpha} s \mid \\
\leq & \frac{\left(d_{2}-d_{1}\right)(1-\lambda)}{\left(d_{2}^{\alpha}-d_{1}^{\alpha}\right)}\left[\int_{0}^{1}\left(\left(z\left(\lambda d_{1}+(1-\lambda) d_{2}\right)+(1-z) d_{1}\right)^{\alpha}-d_{1}^{\alpha}\right) \times\left|\phi^{\prime}\left(z\left(\lambda d_{1}+(1-\lambda) d_{2}\right)+(1-z) d_{1}\right)\right| \mathrm{d} z\right] \\
& +\frac{\left(d_{2}-d_{1}\right) \lambda}{\left(d_{2}^{\alpha}-d_{1}^{\alpha}\right)}\left[\int_{0}^{1}\left(d_{2}^{\alpha}-\left(z d_{2}+(1-z)\left(\lambda d_{1}+(1-\lambda) d_{2}\right)\right)^{\alpha}\right) \times\left|\phi^{\prime}\left(z d_{2}+(1-z)\left(\lambda d_{1}+(1-\lambda) d_{2}\right)\right)\right| \mathrm{d} z\right] \\
\leq & \frac{\left(d_{2}-d_{1}\right)(1-\lambda)}{\left(d_{2}^{\alpha}-d_{1}^{\alpha}\right)}\left[\int_{0}^{1}\left(\left(z\left(\lambda d_{1}+(1-\lambda) d_{2}\right)+(1-z) d_{1}\right)^{\alpha}-d_{1}^{\alpha}\right) \times\left[z\left|\phi^{\prime}\left(\lambda d_{1}+(1-\lambda) d_{2}\right)\right|+(1-z)\left|\phi^{\prime}\left(d_{1}\right)\right|\right] \mathrm{d} z\right] \\
& +\frac{\left(d_{2}-d_{1}\right) \lambda}{\left(d_{2}^{\alpha}-d_{1}^{\alpha}\right)}\left[\int_{0}^{1}\left(d_{2}^{\alpha}-\left(z d_{2}+(1-z)\left(\lambda d_{1}+(1-\lambda) d_{2}\right)\right)^{\alpha}\right) \times\left[z\left|\phi^{\prime}\left(d_{2}\right)\right|+(1-z)\left|\phi^{\prime}\left(\lambda d_{1}+(1-\lambda) d_{2}\right)\right|\right] \mathrm{d} z\right] \\
= & \frac{\left(d_{2}-d_{1}\right)(1-\lambda)}{\left(d_{2}^{\alpha}-d_{1}^{\alpha}\right)}\left[A_{1}(\alpha)\left|\phi^{\prime}\left(d_{1}\right)\right|+B_{1}(\alpha)\left|\phi^{\prime}\left(\lambda d_{1}+(1-\lambda) d_{2}\right)\right|\right] \\
& +\frac{\left(d_{2}-d_{1}\right) \lambda}{\left(d_{2}^{\alpha}-d_{1}^{\alpha}\right)}\left[A_{2}(\alpha)\left|\phi^{\prime}\left(d_{2}\right)\right|+B_{2}(\alpha)\left|\phi^{\prime}\left(\lambda d_{1}+(1-\lambda) d_{2}\right)\right|\right] .
\end{aligned}
$$


Remark 10. Let $\alpha=1$. Then, inequality (39) leads to

$$
\begin{aligned}
& \left|\phi\left(\lambda d_{1}+(1-\lambda) d_{2}\right)-\frac{\alpha}{d_{2}-d_{1}} \int_{d_{1}}^{d_{2}} \phi(s) \mathrm{d} s\right| \\
& \leq(1-\lambda)\left[A_{1}(1)\left|\phi^{\prime}\left(d_{1}\right)\right|+B_{1}(1)\left|\phi^{\prime}\left(\lambda d_{1}+(1-\lambda) d_{2}\right)\right|\right] \\
& \quad+\lambda\left[A_{2}(1)\left|\phi^{\prime}\left(d_{2}\right)\right|+B_{2}(1)\left|\phi^{\prime}\left(\lambda d_{1}+(1-\lambda) d_{2}\right)\right|\right] .
\end{aligned}
$$

\section{Applications to Bivariate Means}

A real-valued function $M:(0, \infty) \times(0, \infty) \longrightarrow(0, \infty)$ is said to be a bivariate mean [82] if $\min \{a, b\} \leq$ $M(a, b) \leq \max \{a, b\}$ for all $a, b \in(0, \infty)$. Recently, the properties and applications for the bivariate means and their related special functions have attracted the attention of many researchers [83-93].

Let $d_{1}, d_{2}>0$ with $d_{1} \neq d_{2}, \alpha \in[-1,0) \cup(0,1]$, and $r \neq 0$. Then, the arithmetic mean $A\left(d_{1}, d_{2}\right)$, logarithmic mean $L\left(d_{1}, d_{2}\right)$, and $(\alpha, r)$-th generalized logarithmic mean $L_{(\alpha, r)}\left(d_{1}, d_{2}\right)$ are defined by

$$
\begin{aligned}
A\left(d_{1}, d_{2}\right) & =\frac{d_{1}+d_{2}}{2}, \\
L\left(d_{1}, d_{2}\right) & =\frac{d_{2}-d_{1}}{\log d_{2}-\log d_{1}}, \\
L_{(\alpha, r)}\left(d_{1}, d_{2}\right) & =\left[\frac{\alpha\left(d_{2}^{r+\alpha}-d_{1}^{r+\alpha}\right)}{\left(d_{2}^{\alpha}-d_{1}^{\alpha}\right)(r+\alpha)}\right]^{1 / r},
\end{aligned}
$$

$$
\begin{aligned}
& \left|A^{r}\left(d_{1}, d_{2}\right)-L_{(\alpha, r)}^{r}\left(d_{1}, d_{2}\right)\right| \\
& \leq \frac{\left(d_{2}-d_{1}\right)(1-\lambda)}{\left(d_{2}^{\alpha}-d_{1}^{\alpha}\right)}\left[\left(A_{1}(\alpha, p)\right)^{1 / p}\left(\frac{\left(\lambda d_{1}+(1-\lambda) d_{2}\right)^{(r-1) q}+d_{1}^{(r-1) q}}{2}\right)^{1 / p}\right] \\
& \quad+\frac{\left(d_{2}-d_{1}\right) \lambda}{\left(d_{2}^{\alpha}-d_{1}^{\alpha}\right)}\left[\left(A_{2}(\alpha, p)\right)^{1 / p}\left(\frac{d_{2}^{(r-1) q}\left(\lambda d_{1}+(1-\lambda) d_{2}\right)^{(r-1) q}}{2}\right)^{1 / q}\right], \\
& \left|A^{-1}\left(d_{1}, d_{2}\right)-L_{(\alpha,-1)}^{-1}\left(d_{1}, d_{2}\right)\right| \\
& \leq \frac{\left(d_{2}-d_{1}\right)(1-\lambda)}{\left(d_{2}^{\alpha}-d_{1}^{\alpha}\right)}\left[\left(A_{1}(\alpha, p)\right)^{1 / p}\left(\frac{\left(\lambda d_{1}+(1-\lambda) d_{2}\right)^{-2 q}+d_{1}^{-2 q}}{2}\right)^{1 / q}\right] \\
& +\frac{\left(d_{2}-d_{1}\right) \lambda}{\left(d_{2}^{\alpha}-d_{1}^{\alpha}\right)}\left[\left(A_{2}(\alpha, p)\right)^{1 / p}\left(\frac{d_{2}^{-2 q}+\left(\lambda d_{1}+(1-\lambda) d_{2}\right)^{-2 q}}{2}\right)^{1 / q}\right],
\end{aligned}
$$

hold for all $\lambda \in[0,1]$, where $A_{1}(\alpha, p)$ and $A_{2}(\alpha, p)$ are defined as in Theorem 6. respectively.

In this section, we shall present several inequalities involving the arithmetic, logarithmic, and generalized logarithmic means.

Let $x>0$ and $\phi(x)=x^{r}$. Then, Theorems 5 and 6 lead to Theorems 11 and 12 immediately.

Theorem 11. Let $d_{1}, d_{2}>0$ with $d_{1}<d_{2}, \quad r>1$, and $\alpha \in(0,1]$. Then, the inequalities

$$
\begin{aligned}
& \left|A^{r}\left(d_{1}, d_{2}\right)-L_{(\alpha, r)}^{r}\left(d_{1}, d_{2}\right)\right| \\
& \leq \frac{\left(d_{2}-d_{1}\right)(1-\lambda)}{\left(d_{2}^{\alpha}-d_{1}^{\alpha}\right)}\left[A_{1}(\alpha) d_{1}^{r-1}+B_{1}(\alpha)\left(\lambda d_{1}+(1-\lambda) d_{2}\right)^{r-1}\right] \\
& \quad+\frac{\left(d_{2}-d_{1}\right) \lambda}{\left(d_{2}^{\alpha}-d_{1}^{\alpha}\right)}\left[A_{2}(\alpha) d_{2}^{r-1}+B_{2}(\alpha)\left(\lambda d_{1}+(1-\lambda) d_{2}\right)^{r-1}\right], \\
& \left|A^{-1}\left(d_{1}, d_{2}\right)-L_{(\alpha,-1)}^{-1}\left(d_{1}, d_{2}\right)\right| \\
& \leq \frac{\left(d_{2}-d_{1}\right)(1-\lambda)}{\left(d_{2}^{\alpha}-d_{1}^{\alpha}\right)}\left[A_{1}(\alpha) d_{1}^{-2}+B_{1}(\alpha)\left(\lambda d_{1}+(1-\lambda) d_{2}\right)^{-2}\right] \\
& +\frac{\left(d_{2}-d_{1}\right) \lambda}{\left(d_{2}^{\alpha}-d_{1}^{\alpha}\right)}\left[A_{2}(\alpha) d_{2}^{-2}+B_{2}(\alpha)\left(\lambda d_{1}+(1-\lambda) d_{2}\right)^{-2}\right],
\end{aligned}
$$

hold for all $\lambda \in[0,1]$, where $A_{1}(\alpha), A_{2}(\alpha), B_{1}(\alpha)$, and $B_{2}(\alpha)$ are defined as in Theorem 5.

Theorem 12. Let $d_{1}, d_{2}>0$ with $d_{1}<d_{2}, r>1, \alpha \in(0,1]$, and $p, q>1$ with $1 / p+1 / q=1$. Then, the inequalities 
Theorem 13. Let $d_{1}, d_{2}>0$ with $d_{1}<d_{2}, \alpha \in(0,1]$, and $q>1$. Then, the inequalities

$$
\begin{aligned}
& \left|A^{r}\left(d_{1}, d_{2}\right)-L_{(\alpha, r)}^{r}\left(d_{1}, d_{2}\right)\right| \\
& \leq \frac{\left(d_{2}-d_{1}\right)(1-\lambda)}{\left(d_{2}^{\alpha}-d_{1}^{\alpha}\right)}\left[\left(M_{1}(\alpha)\right)^{1-(1 / q)}\left(M_{2}(\alpha)\left(\lambda d_{1}+(1-\lambda) d_{2}\right)^{(r-1) q}+M_{3}(\alpha) d_{1}^{(r-1) q}\right)^{1 / q}\right] \\
& +\frac{\left(d_{2}-d_{1}\right) \lambda}{\left(d_{2}^{\alpha}-d_{1}^{\alpha}\right)}\left[\left(N_{1}(\alpha)\right)^{1-(1 / q)}\left(N_{2}(\alpha) d_{2}^{(r-1) q}+N_{3}(\alpha)\left(\lambda d_{1}+(1-\lambda) d_{2}\right)^{(r-1) q}\right)^{1 / q}\right] \\
& \left|A^{-1}\left(d_{1}, d_{2}\right)-L_{(\alpha,-1)}^{-1}\left(d_{1}, d_{2}\right)\right| \\
& \leq \frac{\left(d_{2}-d_{1}\right)(1-\lambda)}{\left(d_{2}^{\alpha}-d_{1}^{\alpha}\right)}\left[\left(M_{1}(\alpha)\right)^{1-(1 / q)}\left(M_{2}(\alpha)\left(\lambda d_{1}+(1-\lambda) d_{2}\right)^{-2 q}+M_{3}(\alpha) d_{1}^{-2 q}\right)^{1 / q}\right] \\
& \left.+\frac{\left(d_{2}-d_{1}\right) \lambda}{d_{2}^{\alpha}-d_{1}^{\alpha}}\left[\left(N_{1}(\alpha)\right)^{1-(1 / q)}\left(N_{2}(\alpha) d_{2}^{-2 q}+N_{3}(\alpha)\left(\lambda d_{1}+(1-\lambda) d_{2}\right)\right)^{-2 q}\right)^{1 / q}\right]
\end{aligned}
$$

hold, where $M_{1}(\alpha), M_{2}(\alpha), M_{3}(\alpha), N_{1}(\alpha), N_{2}(\alpha)$, and $N_{3}(\alpha)$ are defined as in Theorem 8.

\section{Applications to Midpoint Formula}

Let $P$ be the partition of the points $d_{1}=y_{0}<y_{1}<\cdots<$ $y_{n-1}<y_{n}=d_{2}$ of the interval $\left[d_{1}, d_{2}\right]$ and consider the quadrature formula

$$
\int_{d_{1}}^{d_{2}} \phi(y) \mathrm{d}_{\alpha} y=T_{\alpha}(\phi, P)+E_{\alpha}(\phi, P),
$$

where

$$
T_{\alpha}(\phi, P)=\sum_{i=0}^{n-1} \phi\left(\frac{y_{i}+y_{i+1}}{2}\right) \frac{\left(y_{i+1}^{\alpha}-y_{i}^{\alpha}\right)}{\alpha},
$$

is the midpoint version and $E_{\alpha}(\phi, P)$ denotes the associated approximation error. In this section, we are going to derive some new estimates for the midpoint formula.
Theorem 14. Let $d_{1}, d_{2}>0$ with $d_{1}<d_{2}, \alpha \in(0,1]$, and $\phi:\left[d_{1}, d_{2}\right] \longrightarrow \mathbb{R}$ be an $\alpha$-differentiable function. Then, one has

$$
\begin{aligned}
& \left|E_{\alpha}(\phi, P)\right| \\
& \leq \sum_{i=0}^{n-1} \frac{\left(y_{i+1}-y_{i}\right)(1-\lambda)}{\left(y_{i+1}^{\alpha}-y_{i}^{\alpha}\right)}\left[A_{i}(\alpha)\left|\phi^{\prime}\left(y_{i}\right)\right|\right. \\
& \left.\quad+B_{i}(\alpha)\left|\phi^{\prime}\left(\lambda y_{i}+(1-\lambda) y_{i+1}\right)\right|\right] \\
& \quad+\sum_{i=0}^{n-1} \frac{\left(y_{i+1}-y_{i}\right) \lambda}{\left(y_{i+1}^{\alpha}-y_{i}^{\alpha}\right)}\left[\bar{A}_{i}(\alpha)\left|\phi^{\prime}\left(y_{i+1}\right)\right|\right. \\
& \left.\quad+\bar{B}_{i}(\alpha)\left|\phi^{\prime}\left(\lambda y_{i}+(1-\lambda) y_{i+1}\right)\right|\right],
\end{aligned}
$$

if $D_{\alpha}(\phi) \in L_{\alpha}^{1}\left(\left[d_{1}, d_{2}\right]\right)$ and $\left|\phi^{\prime}\right|$ is convex on $\left[d_{1}, d_{2}\right]$, where

$$
\begin{aligned}
& A_{i}(\alpha)=\frac{1}{12}\left[\left(\lambda y_{i}+(1-\lambda) y_{i+1}\right)(\lambda+1)\left(y_{i}^{\alpha-1}+y_{i+1}^{\alpha-1}\right)+\lambda y_{i}\left(y_{i}^{\alpha-1}-y_{i+1}^{\alpha-1}\right)+y_{i}\left(y_{i+1}^{\alpha-1}-5 y_{i}^{\alpha-1}\right)\right] \\
& B_{i}(\alpha)=\frac{\left(\lambda y_{i}+(1-\lambda) y_{i+1}\right)}{12}\left[3 \lambda\left(y_{i}^{\alpha-1}-y_{i+1}^{\alpha-1}\right)+\left(3 y_{i+1}^{\alpha-1}+y_{i}^{\alpha-1}\right)+y_{i}^{\alpha}(\lambda-5)\right] \\
& \bar{A}_{i}(\alpha)=\frac{\lambda\left(y_{i+1}^{\alpha}-y_{i}^{\alpha}\right)}{6} \\
& \bar{B}_{i}(\alpha):=\frac{\lambda\left(y_{i+1}^{\alpha}-y_{i}^{\alpha}\right)}{3} .
\end{aligned}
$$


Proof. Applying Theorem 5 on the subintervals $\left[y_{i}, y_{i+1}\right]$ $(i=0,1, \ldots, n-1)$ of the partition $P$, we have

$$
\begin{aligned}
\mid \phi & \left(\lambda y_{i}+(1-\lambda) y_{i+1}\right) \frac{\left(y_{i+1}^{\alpha}-y_{i}^{\alpha}\right)}{\alpha}-\int_{y_{i}}^{y_{i+1}} \phi(y) \mathrm{d}_{\alpha} y \mid \\
\leq & \frac{\left(y_{i+1}-y_{i}\right)(1-\lambda)}{\left(y_{i+1}^{\alpha}-y_{i}^{\alpha}\right)}\left[A_{i}(\alpha)\left|\phi^{\prime}\left(y_{i}\right)\right|+B_{i}(\alpha)\left|\phi^{\prime}\left(\lambda y_{i}+(1-\lambda) y_{i+1}\right)\right|\right] \\
& +\frac{\left(y_{i+1}-y_{i}\right) \lambda}{\left(y_{i+1}^{\alpha}-y_{i}^{\alpha}\right)}\left[\bar{A}_{i}(\alpha)\left|\phi^{\prime}\left(y_{i+1}\right)\right|+\bar{B}_{i}(\alpha)\left|\phi^{\prime}\left(\lambda y_{i}+(1-\lambda) y_{i+1}\right)\right|\right], \\
\mid \int_{d_{1}}^{d_{2}} & \phi(y) \mathrm{d}_{\alpha} y-T_{\alpha}(\phi, P) \mid \\
= & \left|\sum_{i=0}^{n-1}\left\{\int_{y_{i}}^{y_{i+1}} \phi(y) \mathrm{d}_{\alpha} y-\phi\left(\lambda y_{i}+(1-\lambda) y_{i+1}\right) \frac{\left(y_{i+1}^{\alpha}-y_{i}^{\alpha}\right)}{\alpha}\right\}\right| \\
\leq & \sum_{i=0}^{n-1}\left|\left\{\int_{y_{i}}^{y_{i+1}} \phi(y) \mathrm{d}_{\alpha} y-\phi\left(\lambda y_{i}+(1-\lambda) y_{i+1}\right) \frac{\left(y_{i+1}^{\alpha}-y_{i}^{\alpha}\right)}{\alpha}\right\}\right| \\
\leq & \sum_{i=0}^{n-1} \frac{\left(y_{i+1}-y_{i}\right)(1-\lambda)}{\left(y_{i+1}^{\alpha}-y_{i}^{\alpha}\right)}\left[A_{i}(\alpha)\left|\phi^{\prime}\left(y_{i}\right)\right|+B_{i}(\alpha)\left|\phi^{\prime}\left(\lambda y_{i}+(1-\lambda) y_{i+1}\right)\right|\right] \\
& +\sum_{i=0}^{n-1} \frac{\left(y_{i+1}-y_{i}\right) \lambda}{\left(y_{i+1}^{\alpha}-y_{i}^{\alpha}\right)}\left[\bar{A}_{i}(\alpha)\left|\phi^{\prime}\left(y_{i+1}\right)\right|+\bar{B}_{i}(\alpha)\left|\phi^{\prime}\left(\lambda y_{i}+(1-\lambda) y_{i+1}\right)\right|\right] .
\end{aligned}
$$

Making use of Theorems 6-8 and similar arguments as in the proof of Theorem 14, we get Theorems 15 and 16 immediately.

Theorem 15. Let $d_{1}, d_{2}>0$ with $d_{1}<d_{2}, \alpha \in(0,1]$, and $\phi:\left[d_{1}, d_{2}\right] \longrightarrow \mathbb{R}$ be an $\alpha$-differentiable function and $p, q>1$ with $1 / p+1 / q=1$. Then, the inequality

$$
\begin{aligned}
\left|E_{\alpha}(\phi, P)\right| \leq & \sum_{i=0}^{n-1} \frac{\left(y_{i+1}-y_{i}\right)(1-\lambda)}{\left(y_{i+1}^{\alpha}-y_{i}^{\alpha}\right)}\left[\left(A_{i}(\alpha, p)\right)^{1 / p} E_{i}^{1 / q}\right] \\
& +\sum_{i=0}^{n-1} \frac{\left(y_{i+1}-y_{i}\right) \lambda}{\left(y_{i+1}^{\alpha}-y_{i}^{\alpha}\right)}\left[\left(\bar{A}_{i}(\alpha, p)\right)^{1 / p} \bar{E}_{i}^{1 / q}\right],
\end{aligned}
$$

holds for all $\lambda \in[0,1]$ if $D_{\alpha}(\phi) \in L_{\alpha}^{1}\left(\left[d_{1}, d_{2}\right]\right)$ and $\left|\phi^{\prime}\right|^{q}$ is convex on $\left[d_{1}, d_{2}\right]$, where

$$
\begin{aligned}
E_{i}(\alpha) & =\frac{\left|\phi^{\prime}\left(\lambda y_{i}+(1-\lambda) y_{i+1}\right)\right|^{q}+\left|\phi^{\prime}\left(y_{i}\right)\right|^{q}}{2}, \\
E_{i}(\alpha) & =\frac{\left|\phi^{\prime}\left(y_{i+1}\right)\right|^{q}+\left|\phi^{\prime}\left(\lambda y_{i}+(1-\lambda) y_{i+1}\right)\right|^{q}}{2}, \\
A_{i}(\alpha, p) & =\frac{1}{(\lambda-1)\left(y_{i}-y_{i+1}\right)} \int_{y_{i}}^{\lambda y_{i}+(1-z) y_{i+1}}\left(s^{\alpha}-y_{i}^{\alpha}\right)^{p} \mathrm{~d} s, \\
\bar{A}_{i}(\alpha, p) & =\frac{1}{\lambda\left(y_{i+1}-y_{i}\right)} \int_{\lambda y_{i}+(1-z) y_{i+1}}^{y_{i+1}}\left(y_{i+1}^{\alpha}-s^{\alpha}\right)^{p} \mathrm{~d} s .
\end{aligned}
$$

Theorem 16. Let $d_{1}, d_{2}>0$ with $d_{1}<d_{2}, \alpha \in(0,1]$, and $\phi:\left[d_{1}, d_{2}\right] \longrightarrow \mathbb{R}$ be an $\alpha$-differentiable function and $q \geq 1$. Then, the inequality

$$
\begin{aligned}
& \left|E_{\alpha}(\phi, P)\right| \\
& \quad \leq \sum_{i=0}^{n-1} \frac{\left(y_{i+1}-y_{i}\right)(1-\lambda)}{\left(y_{i+1}^{\alpha}-y_{i}^{\alpha}\right)}\left[\left(M_{i}(\alpha)\right)^{1-(1 / q)}\left(\bar{M}_{i}(\alpha)\left|\phi^{\prime}\left(\lambda y_{i}+(1-\lambda) y_{i+1}\right)\right|^{q}+\bar{M}_{i}(\alpha)\left|\phi^{\prime}\left(y_{i}\right)\right|^{q}\right)^{1 / q}\right] \\
& \quad+\sum_{i=0}^{n-1} \frac{\left(y_{i+1}-y_{i}\right) \lambda}{\left(y_{i+1}^{\alpha}-y_{i}^{\alpha}\right)}\left[\left(N_{i}(\alpha)\right)^{1-(1 / q)}\left(\bar{N}_{i}(\alpha)\left|\phi^{\prime}\left(y_{i+1}\right)\right|^{q}+\bar{N}_{i}(\alpha)\left|\phi^{\prime}\left(\lambda y_{i}+(1-\lambda) y_{i+1}\right)\right|^{q}\right)^{1 / q}\right],
\end{aligned}
$$


holds for all $\lambda \in[0,1]$ if $D_{\alpha}(\phi) \in L_{\alpha}^{1}\left(\left[d_{1}, d_{2}\right]\right)$ and $\left|\phi^{\prime}\right|^{q}$ is convex on $\left[d_{1}, d_{2}\right]$, where

$$
\begin{aligned}
& M_{i}(\alpha)=\left[\frac{y_{i}^{\alpha}\left(\lambda^{2}+\lambda-2\right)+y_{i+1}^{\alpha}(\lambda-1)^{2}}{3}-\frac{y_{i+1} y_{i}^{\alpha-1}\left(2 \lambda^{2}+\lambda-3\right)+y_{i} y_{i+1}^{\alpha-1}\left(2 \lambda^{2}-\lambda-1\right)}{6}\right], \\
& N_{i}(\alpha)=\frac{\lambda\left(y_{i+1}^{\alpha}-y_{i}^{\alpha}\right)}{2}=3 \bar{N}_{i}(\alpha)=\frac{3}{2} \bar{N}_{i}(\alpha), \\
& \bar{M}_{i}(\alpha)=\frac{(\lambda-1)\left(y_{i}^{\alpha}-y_{i+1}^{\alpha}\right)}{3}=2 \bar{M}_{i}(\alpha) .
\end{aligned}
$$

\section{Data Availability}

No data were used to support this study.

\section{Conflicts of Interest}

The authors declare that there are no conflicts of interest regarding the publication of this paper.

\section{Authors' Contributions}

All authors contributed equally to writing of this paper. All authors read and approved the final manuscript.

\section{Acknowledgments}

This work was supported by the Natural Science Foundation of China (grant nos. 61673169, 11971142, 11871202, 11301127, 11701176, 11626101, and 11601485).

\section{References}

[1] J. E. Pečarić, F. Proschan, and Y. L. Tong, Convex Functions, Partial Orderings, and Statistical Applications, Academic Press, Boston, MA, USA, 1992.

[2] T.-H. Zhao, Y.-M. Chu, and H. Wang, "Logarithmically complete monotonicity properties relating to the gamma function," Abstract and Applied Analysis, vol. 2011, Article ID 896483, 13 pages, 2011.

[3] Z. Dai and F. Wen, "Another improved Wei-Yao-Liu nonlinear conjugate gradient method with sufficient descent property," Applied Mathematics and Computation, vol. 218, no. 14, pp. 7421-7430, 2012.

[4] Y. Jiang and J. Ma, "Spectral collocation methods for volterraintegro differential equations with noncompact kernels," Journal of Computational and Applied Mathematics, vol. 244, pp. 115-124, 2013.

[5] Z.-F. Dai and F.-H. Wen, "Robust CVaR-based portfolio optimization under a genal affine data perturbation uncertainty set," Journal of Computational Analysis \& Applications, vol. 16, no. 1, pp. 93-103, 2014.

[6] C. Huang, S. Guo, and L. Liu, "Boundedness on Morrey space for Toeplitz type operator associated to singular integral operator with variable Calderón-Zygmund kernel," Journal of Mathematical Inequalities, vol. 8, no. 3, pp. 453-464, 2014.
[7] W. Zhou and F. Wang, "A PRP-based residual method for large-scale monotone nonlinear equations," Applied Mathematics and Computation, vol. 261, pp. 1-7, 2015.

[8] X.-P. Fang, Y.-J. Deng, and J. Li, "Plasmon resonance and heat generation in nanostructures," Mathematical Methods in the Applied Sciences, vol. 38, no. 18, pp. 4663-4672, 2016.

[9] J. Li, G. Sun, and R. Zhang, "The numerical solution of scattering by infinite rough interfaces based on the integral equation method," Computers \& Mathematics with Applications, vol. 71, no. 7, pp. 1491-1502, 2016.

[10] M. K. Wang, Y. M. Chu, and Y. P. Jiang, "Ramanujan's cubic transformation inequalities for zero-balanced hypergeometric functions," Rocky Mountain Journal of Mathematics, vol. 46, no. 2, pp. 679-691, 2016.

[11] H.-H. Chu, Z.-H. Yang, Y.-M. Chu, and W. Zhang, "Generalized Wilker-type inequalities with two parameters," Journal of Inequalities and Applications, vol. 2016, Article ID 187, 13 pages, 2016.

[12] L. Duan and C. Huang, "Existence and global attractivity of almost periodic solutions for a delayed differential neoclassical growth model," Mathematical Methods in the Applied Sciences, vol. 40, no. 3, pp. 814-822, 2017.

[13] C. Liu, Z. Gong, K. L. Teo, J. Sun, and L. Caccetta, "Robust multi-objective optimal switching control arising in 1,3propanediol microbial fed-batch process," Nonlinear Analysis: Hybrid Systems, vol. 25, pp. 1-20, 2017.

[14] W. Wang, "On A-stable one-leg methods for solving nonlinear Volterra functional differential equations," Applied Mathematics and Computation, vol. 314, pp. 380-390, 2017.

[15] Y. Tan, C. Huang, B. Sun, and T. Wang, "Dynamics of a class of delayed reaction-diffusion systems with Neumann boundary condition," Journal of Mathematical Analysis and Applications, vol. 458, no. 2, pp. 1115-1130, 2018.

[16] T.-R. Huang, B.-W. Han, X.-Y. Ma, and Y.-M. Chu, "Optimal bounds for the generalized Euler-Mascheroni constant," Journal of Inequalities and Applications, vol. 2018, p. 9, 2018.

[17] Z. Liu, N. Wu, X. Qin, and Y. Zhang, "Trigonometric transform splitting methods for real symmetric Toeplitz systems," Computers \& Mathematics with Applications, vol. 75, no. 8, pp. 2782-2794, 2018.

[18] T.-R. Huang, S.-Y. Tan, X.-Y. Ma, and Y.-M. Chu, "Monotonicity properties and bounds for the complete $p$-elliptic integrals," Journal of Inequalities and Applications, vol. 2018, p. 11, 2018.

[19] J. Wang, X. Chen, and L. Huang, "The number and stability of limit cycles for planar piecewise linear systems of node-saddle type," Journal of Mathematical Analysis and Applications, vol. 469, no. 1, pp. 405-427, 2019. 
[20] M.-K. Wang, Y.-M. Chu, and W. Zhang, "Monotonicity and inequalities involving zero-balanced hypergeometric function," Mathematical Inequalities \& Applications, vol. 22, no. 2, pp. 601-617, 2019.

[21] J. Peng and Y. Zhang, "Heron triangles with figurate number sides," Acta Mathematica Hungarica, vol. 157, no. 2, pp. 478-488, 2019.

[22] C. Huang, H. Zhang, and L. Huang, "Almost periodicity analysis for a delayed Nicholson's blowflies model with nonlinear density-dependent mortality term," Communications on Pure \& Applied Analysis, vol. 18, no. 6, pp. 3337-3349, 2019.

[23] M.-K. Wang, W. Zhang, and Y.-M. Chu, "Monotonicity, convexity and inequalities involving the generalized elliptic integrals," Acta Mathematica Scientia, vol. 39B, no. 5, pp. 1440-1450, 2019.

[24] Z. Liu, S. Chen, W. Xu, and Y. Zhang, "The eigen-structures of real (skew) circulant matrices with some applications," Computational and Applied Mathematics, vol. 38, no. 4, p. 13, 2019.

[25] M.-K. Wang, H.-H. Chu, and Y.-M. Chu, "Precise bounds for the weighted Hölder mean of the complete p-elliptic integrals," Journal of Mathematical Analysis and Applications, vol. 480, no. 2, Article ID 123388, 2019.

[26] N. Zhu, Z. Liu, F. Wang, and K. Zhao, "Explicit decay rates for a generalized Boussinesq-Burgers system," Applied Mathematics Letters, vol. 100, Article ID 106054, 2020.

[27] J.-F. Tian, Y.-R. Zhu, and W.-S. Cheung, "n-tuple diamondalpha integral and inequalities on time scales," Revista de la Real Academia de Ciencias Exactas, Físicas y Naturales. Serie A. Matemáticas, vol. 113, no. 3, pp. 2189-2200, 2019.

[28] W. Zhou, "On the convergence of the modified LevenbergMarquardt method with a nonmonotone second order Armijo type line search," Journal of Computational and Applied Mathematics, vol. 239, pp. 152-161, 2013.

[29] W. Zhou and X. Chen, "On the convergence of a modified regularized Newton method for convex optimization with singular solutions," Journal of Computational and Applied Mathematics, vol. 239, pp. 179-188, 2013.

[30] L. Zhang and S. Jian, "Further studies on the Wei-Yao-Liu nonlinear conjugate gradient method," Applied Mathematics and Computation, vol. 219, no. 14, pp. 7616-7621, 2013.

[31] X.-F. Li, G.-J. Tang, and B.-Q. Tang, "Stress field around a strike-slip fault in orthotropic elastic layers via a hypersingular integral equation," Computers \& Mathematics with Applications, vol. 66, no. 11, pp. 2317-2326, 2013.

[32] G.-X. Qin, C.-X. Huang, Y.-Q. Xie, and F.-H. Wen, "Asymptotic behavior for third-order quasi-linear differential equations," Advances in Difference Equations, vol. 2013, p. 8, 2013.

[33] C. Huang, Z. Yang, T. Yi, and X. Zou, "On the basins of attraction for a class of delay differential equations with nonmonotone bistable nonlinearities," Journal of Differential Equations, vol. 256, no. 7, pp. 2101-2114, 2014.

[34] Y. Liu and J. Wu, "Fixed point theorems in piecewise continuous function spaces and applications to some nonlinear problems," Mathematical Methods in the Applied Sciences, vol. 37, no. 4, pp. 508-517, 2014.

[35] W. Tang and Y. Sun, "Construction of Runge-Kutta type methods for solving ordinary differential equations," Applied Mathematics and Computation, vol. 234, pp. 179-191, 2014.

[36] D. Xie and J. Li, "A new analysis of electrostatic free energy minimization and Poisson-Boltzmann equation for protein in ionic solvent," Nonlinear Analysis: Real World Applications, vol. 21, pp. 185-196, 2015.
[37] X.-S. Zhou, "Weighted sharp function estimate and boundedness for commutator associated with singular integral operator satisfying a variant of Hörmander's condition," Journal of Mathematical Inequalities, vol. 9, no. 2, pp. 587596, 2015.

[38] Z. Dai, X. Chen, and F. Wen, “A modified Perry's conjugate gradient method-based derivative-free method for solving large-scale nonlinear monotone equations," Applied Mathematics and Computation, vol. 270, pp. 378-386, 2015.

[39] Z. Dai, "Comments on a new class of nonlinear conjugate gradient coefficients with global convergence properties," Applied Mathematics and Computation, vol. 276, pp. 297-300, 2016.

[40] Z.-F. Dai, D.-H. Li, and F.-H. Wen, "Worse-case conditional value-at-risk for asymmetrically distributed asset scenarios returns," Journal of Computational Analysis and Applications, vol. 20, no. 2, pp. 237-251, 2016.

[41] Y. Tan and K. Jing, "Existence and global exponential stability of almost periodic solution for delayed competitive neural networks with discontinuous activations," Mathematical Methods in the Applied Sciences, vol. 39, no. 11, pp. 2821-2839, 2016.

[42] L. Duan, L. Huang, Z. Guo, and X. Fang, "Periodic attractor for reaction-diffusion high-order Hopfield neural networks with time-varying delays," Computers \& Mathematics with Applications, vol. 73, no. 2, pp. 233-245, 2017.

[43] W. Wang and Y. Chen, "Fast numerical valuation of options with jump under Merton's model," Journal of Computational and Applied Mathematics, vol. 318, pp. 79-92, 2017.

[44] C. Huang and L. Liu, "Boundedness of multilinear singular integral operator with non-smooth kernels and mean oscillation," Quaestiones Mathematicae, vol. 40, no. 3, pp. 295-312, 2017.

[45] H.-J. Hu and L.-Z. Liu, "Weighted inequalities for a general commutator associated to a singular integral operator satisfying a variant of Hörmander's condition," Mathematical Notes, vol. 101, no. 5-6, pp. 830-840, 2017.

[46] Z. Cai, J.-H. Huang, and L. Huang, "Generalized LyapunovRazumikhin method for retarded differential inclusions: applications to discontinuous neural networks," Discrete \& Continuous Dynamical Systems-B, vol. 22, no. 9, pp. 35913614, 2017.

[47] $\mathrm{H}$. Hu and X. Zou, "Existence of an extinction wave in the Fisher equation with a shifting habitat," Proceedings of the American Mathematical Society, vol. 145, no. 11, pp. 47634771, 2017.

[48] Z. Liu, X. Qin, N. Wu, and Y. Zhang, "The shifted classical circulant and skew circulant splitting iterative methods for Toeplitz matrices," Canadian Mathematical Bulletin, vol. 60, no. 4, pp. 807-815, 2017.

[49] W. Tang and J. Zhang, "Symplecticity-preserving continuousstage Runge-Kutta-Nyström methods," Applied Mathematics and Computation, vol. 323, pp. 204-219, 2018.

[50] L. Duan, X. Fang, and C. Huang, "Global exponential convergence in a delayed almost periodic Nicholson's blowflies model with discontinuous harvesting," Mathematical Methods in the Applied Sciences, vol. 41, no. 5, pp. 1954-1965, 2018.

[51] C.-X. Huang, Y.-C. Qiao, L.-H. Huang, and R. P. Agarwal, "Dynamical behaviors of a food-chain model with stage structure and time delays," Advances in Difference Equations, vol. 2018, p. 26, 2018.

[52] K. X. Zhu, Y. Q. Xie, and F. Zhou, "Pullback attractors for a damped semilinear wave equation with delays," Acta Mathematica Sinica, English Series, vol. 34, no. 7, pp. 1131-1150, 2018. 
[53] Z. Cai, J. Huang, and L. Huang, "Periodic orbit analysis for the delayed Filippov system," Proceedings of the American Mathematical Society, vol. 146, no. 11, pp. 4667-4682, 2018.

[54] J. Li, J. Ying, and D. Xie, "On the analysis and application of an ion size-modified Poisson-Boltzmann equation," Nonlinear Analysis: Real World Applications, vol. 47, pp. 188-203, 2019.

[55] J. Wang, C. Huang, and L. Huang, "Discontinuity-induced limit cycles in a general planar piecewise linear system of saddle-focus type," Nonlinear Analysis: Hybrid Systems, vol. 33, pp. 162-178, 2019.

[56] Y. Jiang and $\mathrm{X}$. Xu, "A monotone finite volume method for time fractional Fokker-Planck equations," Science China Mathematics, vol. 62, no. 4, pp. 783-794, 2019.

[57] Z. Tian, Y. Liu, Y. Zhang, Z. Liu, and M. Tian, "The general inner-outer iteration method based on regular splittings for the PageRank problem," Applied Mathematics and Computation, vol. 356, pp. 479-501, 2019.

[58] W. Wang, Y. Chen, and H. Fang, "On the variable two-step IMEX BDF method for parabolic integro-differential equations with nonsmooth initial data arising in finance," SIAM Journal on Numerical Analysis, vol. 57, no. 3, pp. 1289-1317, 2019.

[59] C.-X. Huang, H. Zhang, J.-D. Cao, and H.-J. Hu, "Stability and Hopf bifurcation of a delayed prey-predator model with disease in the predator," International Journal of Bifurcation and Chaos, vol. 29, no. 7, p. 23, 2019.

[60] F. Liu, L. Feng, V. Anh, and J. Li, "Unstructured-mesh Galerkin finite element method for the two-dimensional multi-term time-space fractional Bloch-Torrey equations on irregular convex domains," Computers \& Mathematics with Applications, vol. 78, no. 5, pp. 1637-1650, 2019.

[61] T. Wang and H. Guo, "Existence and nonexistence of nodal solutions for Choquard type equations with perturbation," Journal of Mathematical Analysis and Applications, vol. 480, no. 2, Article ID 123438, 2019.

[62] K.-X. Zhu, Y.-Q. Xie, and F. Zhou, " $L^{p}$-pullback attractors for non-autonomous reaction-diffusion equations with delays," Topological Methods in Nonlinear Analysis, vol. 54, no. 1, pp. 9-27, 2019.

[63] M. Li and T. Sugawa, "Schur parameters and the carathéodory class," Results in Mathematics, vol. 74, no. 4, p. 13, 2019.

[64] X. Long and S. Gong, "New results on stability of Nicholson's blowflies equation with multiple pairs of time-varying delays," Applied Mathematics Letters, vol. 100, Article ID 106027, 6 pages, 2020.

[65] Y.-M. Chu, M. Adil Khan, T. Ali, and S. S. Dragomir, "Inequalities for $\alpha$-fractional differentiable functions," Journal of Inequalities and Applications, vol. 2017, p. 12, 2017.

[66] Y.-Q. Song, M. Adil Khan, S. Zaheer Ullah, and Y.-M. Chu, "Integral inequalities involving strongly convex functions," Journal of Function Spaces, vol. 2018, Article ID 6595921, 8 pages, 2018.

[67] M. Adil Khan, S.-H. Wu, H. Ullah, and Y.-M. Chu, "Discrete majorization type inequalities for convex functions on rectangles," Journal of Inequalities and Applications, vol. 2019, p. 18, 2019.

[68] S. Zaheer Ullah, M. Adil Khan, and Y.-M. Chu, "Majorization theorems for strongly convex functions," Journal of Inequalities and Applications, vol. 2019, p. 13, 2019.

[69] S. Zaheer Ullah, M. Adil Khan, Z. A. Khan, and Y.-M. Chu, "Integral majorization type inequalities for the functions in the sense of strong convexity," Journal of Function Spaces, vol. 2019, Article ID 9487823, 11 pages, 2019.

[70] M. Adil Khan, S. Zaheer Ullah, and Y.-M. Chu, "The concept of coordinate strongly convex functions and related inequalities," Revista de la Real Academia de Ciencias Exactas, Físicas y Naturales: Serie A-Matemáticas, vol. 113, no. 2, pp. 2235-2251, 2019.

[71] M. Adil Khan, M. Hanif, Z. A. Khan, K. Ahmad, and Y.-M. Chu, "Association of Jensen's inequality for s-convex function with Csiszár divergence," Journal of Inequalities and Applications, vol. 2019, p. 14, 2019.

[72] I. Abbas Baloch and Y.-M. Chu, "Petrović-type inequalities for harmonic h-convex functions," Journal of Function Spaces, vol. 2020, Article ID 3075390, 7 pages, 2020.

[73] J. Hadamard, "Étude sur les propriétés des fonctions entières et en particulier dune fonction considérée par Riemann," Journal de Mathématiques Pures et Appliquées, vol. 58, pp. 171-215, 1893.

[74] M. Adil Khan, A. Iqbal, M. Suleman, and Y.-M. Chu, "Hermite-Hadamard type inequalities for fractional integrals via Green's function," Journal of Inequalities and Applications, vol. 2018, p. 15, 2018.

[75] M. Adil Khan, Y. Khurshid, T.-S. Du, and Y.-M. Chu, "Generalization of Hermite-Hadamard type inequalities via conformable fractional integrals," Journal of Function Spaces, vol. 2018, Article ID 5357463, 12 pages, 2018.

[76] S. Rashid, M. A. Noor, K. I. Noor, F. Safdar, and Y.-M. Chu, "Hermite-Hadamard type inequalities for the class of convex functions on time scale," Mathematics, vol. 7, no. 10, Article ID 956, 2019.

[77] S. Zaheer Ullah, M. Adil Khan, and Y.-M. Chu, "A note on generalized convex functions," Journal of Inequalities and Applications, vol. 2019, p. 10, 2019.

[78] M. W. Alomari, M. Darus, and U. S. Kirmaci, "Some inequalities of Hermite-Hadamard type for s-convex functions," Acta Mathematica Scientia, vol. 31, no. 4, pp. 1643-1652, 2011.

[79] F.-X. Chen and Y.-M. Feng, "New inequalities of HermiteHadamard type for functions whose first derivatives absolute values are s-convex," Italian Journal of Pure and Applied Mathematics, vol. 32, pp. 213-222, 2014.

[80] R. Khalil, M. Al Horani, A. Yousef, and M. Sababheh, "A new definition of fractional derivative," Journal of Computational and Applied Mathematics, vol. 264, pp. 65-70, 2014.

[81] D. R. Anderson, “Taylor's formula and integral inequalities for conformable fractional derivatives," in Contributions in Mathematics and Engineering, vol. 25-43, Cham, Switzerland, Springer, 2016.

[82] P. S. Bullen, D. S. Mitrinović, and P. M. Vasić, Means and Their Inequalities, D. Reidel Publishing Co., Dordrecht, Netherlands, 1988.

[83] Y.-M. Chu, M.-K. Wang, and S.-L. Qiu, "Optimal combinations bounds of root-square and arithmetic means for Toader mean," Proceedings-Mathematical Sciences, vol. 122, no. 1, pp. 41-51, 2012.

[84] Y.-M. Chu, H. Wang, and T.-H. Zhao, "Sharp bounds for the Neuman mean in terms of the quadratic and second Seiffert means," Journal of Inequalities and Applications, vol. 2014, p. 14, 2014.

[85] H. Wang, W.-M. Qian, and Y.-M. Chu, "Optimal bounds for Gaussian arithmetic-geometric mean with applications to complete elliptic integral," Journal of Function Spaces, vol. 2016, Article ID 3698463, 6 pages, 2016.

[86] W.-M. Qian, X. Zhang, and Y. Chu, "Sharp bounds for the Toader-Qi mean in terms of harmonic and geometric means," Journal of Mathematical Inequalities, vol. 11, no. 1, pp. 121127, 2017

[87] H.-Z. Xu, Y.-M. Chu, and W.-M. Qian, "Sharp bounds for the Sándor-Yang means in terms of arithmetic and contra- 
harmonic means," Journal of Inequalities and Applications, vol. 2018, p. 13, 2018.

[88] S.-H. Wu and Y.-M. Chu, "Schur $m$-power convexity of generalized geometric Bonferroni mean involving three parameters," Journal of Inequalities and Applications, vol. 2019, p. 11, 2019.

[89] W.-M. Qian, H.-Z. Xu, and Y.-M. Chu, "Improvements of bounds for the Sándor-Yang means," Journal of Inequalities and Applications, vol. 2019, p. 8, 2019.

[90] X.-H. He, W.-M. Qian, H.-Z. Xu, and Y.-M. Chu, "Sharp power mean bounds for two Sándor-Yang means," Revista de la Real Academia de Ciencias Exactas, Físicas y Naturales: Serie A-Matemáticas, vol. 113, no. 3, pp. 2627-2638, 2019.

[91] W.-M. Qian, Z.-Y. He, H.-W. Zhang, and Y.-M. Chu, "Sharp bounds for Neuman means in terms of two-parameter contraharmonic and arithmetic mean," Journal of Inequalities and Applications, vol. 2019, p. 13, 2019.

[92] W.-M. Qian, Y.-Y. Yang, H.-W. Zhang, and Y.-M. Chu, "Optimal two-parameter geometric and arithmetic mean bounds for the Sándor-Yang mean," Journal of Inequalities and Applications, vol. 2019, p. 12, 2019.

[93] B. Wang, C.-L. Luo, S.-H. Li, and Y.-M. Chu, "Sharp oneparameter geometric and quadratic means bounds for the Sándor-Yang means," Revista de la Real Academia de Ciencias Exactas, Físicas y Naturales: Serie A-Matemáticas, vol. 114, no. $1,2020$. 